UCRL-ID 112778

Reprinted from Engineering Research, Development, and Technology.

Thrust Area Report FY 92

UCRL 53868-92

\title{
Remote Sensing, Imaging, and Signal Engineering
}

\section{James M. Brase}

March, 1993

This is an informal report intended primarily for internal or limited external distribution. The opinions and conclusions stated are those of the author and may or may not be those of the Laboratory.

- Work performed under the auspices of the U.S. Department of Energy by the Lawrence Livermore National Laboratory under Contract W-7405-Eng-48. 


\section{Disclaimer}

This document was prepared as an account of work sponsored by an agency of the United States Government. Neither the United States Government nor the University of California nor any of their employees, makes any warranty, express or implied, or assumes any legal liability or responsibility for the accuracy, completeness, or usefulness of any information, apparatus, product, or process disclosed, or represents that its use would not infringe privately owned rights. Reference herein to any specific commercial products, process, or service by trade name, trademark, manufacturer, or otherwise, does not necessarily constitute or imply its endorsement, recommendation, or favoring by the United States Government or the University of California. The views and opinions of authors expressed herein do not necessarily state or reflect those of the United States Government or the University of California and shall not be used for advertising or product endorsement purposes.

This report has been reproduced directly from the best available copy.

Available to DOE and DOE contractors from the Office of Scientific and Technical Information P.O. Box 62, Oak Ridge, TN 37831

Prices available from (615) 576-8401, FTS 626-8401

Available to the public from the National Technical Information Service

U.S. Department of Commerce

5285 Port Royal Rd.,

Springfield, VA 22161 


\title{
Remote Sensing, Imaging, and Signal Engineering
}

\author{
James M. Brase
}




\section{Remote Sensing, Imaging, and Signal Engineering}

Signal and image processing have always been important support for existing programs at Lawrence Livermore National Laboratory (LLNL), but now these technologies are becoming central to the formation of new programs. Exciting new applications such as high-resolution telescopes, radar remote sensing, and advanced medical imaging are allowing us to participate in the development of new programs.

The Remote Sensing, Imaging, and Signal Engineering (RISE) thrust area has been very active in working to define new directions.

We also maintain and continue to build our technical base in signal and image processing in support of existing programs, through such applications as diagnostic image processing and seismic signal processing.

Over the past several years, RISE has developed a series of computer software systems for signal and image processing. These systems provide portability among the many computer systems used at LLNL and give us a platform for transferring the results of specific research and development projects to application areas. Our major signal-and image-processing systems, VIEW and VISION, are used by several major LLNL programs and have been distributed to many university, industry, and government sites.

Work in RISE involves a diverse set of sciences and technologies ranging from optical physics to microbiology to advanced computer architectures. Collaboration with other thrust areas, such as Nondestructive Evaluation and Computational Electronics and Electromagnetics, and with other LLNL organizations, such as the Physics Department and the Biomedical Sciences Division, is central to our continuing work in innovative imaging and signal-processing applications. 


\section{Section 9}

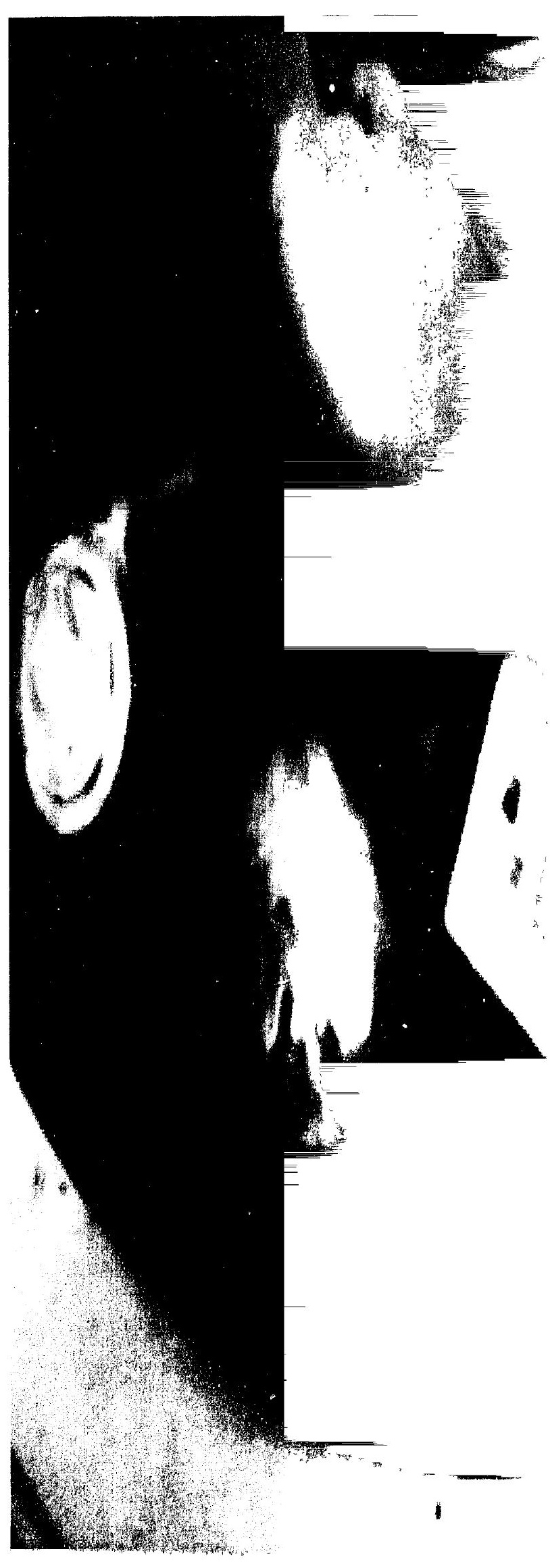




\section{Remote Sensing, Imaging, and Signal Engineering}

Overview

James M. Brase, Thrust Aren Leader

Vision-Based Grasping for Autonomous Sorting of Unknown Objects

Shin-yee Lu, Robert K. Johnson, and Jose E. Hernandez

Image-Restoration and Image-Recovery Algorithms

Dennis M. Goodman

View: A Signal- and Image-Processing System

James M. Brase, Sean K. Lehman, Melvin G. Wieting,

Joseph P. Phillips, and Hanna Szoke

VISION: An Object-Oriented Environment for Computer Vision and Pattern Recognition

Jose E. Hernandez and Michael R. Buhl

Biomedical Image Processing

Laura N. Mascio

\section{Multisensor Data Fusion Using Fuzzy Logic}

Donald T. Gavel

\section{Adaptive Optics for Laser Guide Stars}

James M. Brase, Kenneth Avicola, Donald T. Gavel,

Kenneth E. Waltjen, and Horst D. Bissinger 


\title{
Vision-Based Grasping for Autonomous Sorting of Unknown Objects
}

\author{
Shin-yee Lu, \\ Robert K. Johnson, and \\ Jose E. Hemandez \\ Engineering Research Division \\ Electronics Engineering
}

The Department of Energy has a need for a method of treating existing nuclear waste. Hazardous waste stored in drums and boxes in old warehouses needs to be sorted and treated by the new standards of environmental regulations. At Lawrence Livermore National Laboratory, we are developing a vision-based grasping capability that can be used to pick and place unknown objects autonomously. Some preliminary results are described in this paper.

\section{Introduction}

In our experiment, we lay several objects on a table at arbitrary locations, simulating a conveyor belt. The objects are wrapped in plastic bags to simulate articles that are likely to be found in the waste containers. Twocameras are mounted above the table to create a stereo view of the work cell. The cameras are mounted approximately $2 \mathrm{~m}$ above the table, and have a field of view of approximatel $2 \mathrm{~m}$ by $2 \mathrm{~m}$. The images are captured and processed on a SUN SPARCStation-2, with image resolution of $510 \times 480$.

The stereo images are registered pixel-by-pixel using an efficient stereo-vision algorithm. A dense, three-dimensional (3-D) range map is generated by triangulating the registered pixels. Potential grasp locations for each object are generated by analyzing the shape of a two-dimensional (2-D) projection of the top view of the object. Locations around the handle or near the center of mass of the objex. are considered suitable for grasping, using a parallel gripper. The result of this analysis is used to generate information such as position, height, width, and orientation for executing the grasping task.

\section{Progress}

The experimental result shows high accuracy in the range estimation. We videotaped the experiment and studied the performance. The overall accuracy on the plane perpendicular to the camer- as' lines of sight is within $2 \mathrm{~mm}$, and along the line of sight is $5 \mathrm{~mm}$. The total CPU time required for generating the grasping information is approximateiy $70 \mathrm{~s}$ for four objects. The computation time is proportional to the number of objects to be handled.

This experiment is an integration of camera calibration, stereo registration, shape analysis, and grasp planning. Algorithms used for camera calibration and image segmentation follow existing methods; however, our approach to stereo registration is different from most of the existing methods. It is efficient and highly parallelizable. Grasp planning at this point is a simple decision tree that matches the dynamic range of grippers to the size of the objects. Each of the different tasks is explained below in more detail, with an emphasis on the stereo registration algorithm.

\section{General Approach}

A set of transformation matrices for epipolar geometry correction are obtained through a camera calibration procedure. The images are segmented into regions, using a thresholding technique that separates the objects from the background. Since we assume that the objects are not touching each other, each region segment is assumed to correspond to an object in the scene. Correspondence of regions from the left image with those from the right image is then established, using features such as location and size of 


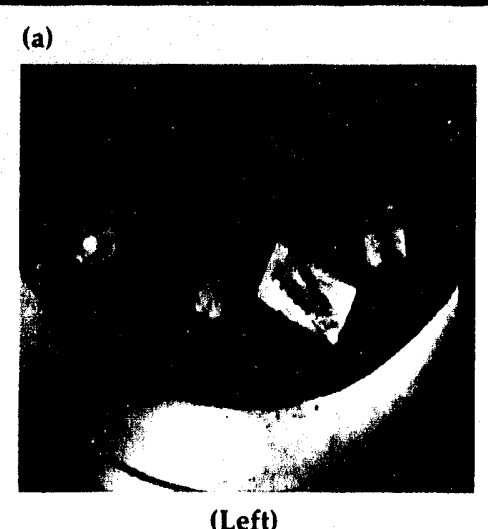

(b)

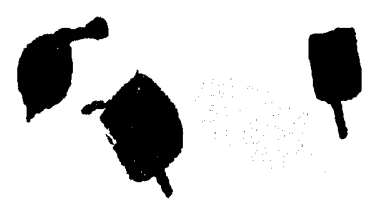

(Left)

Figure 1. (a) Left and right images of a typical scene with four objects on a table: (b) segmented images with, corresponded regions.

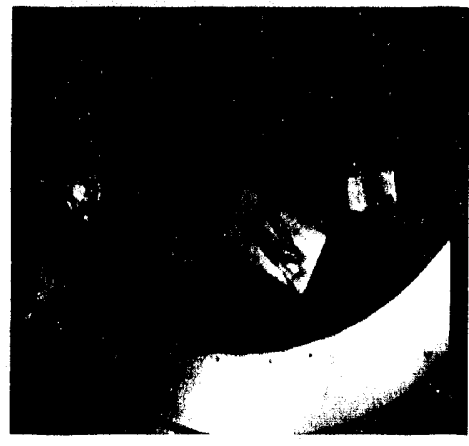

(Right)
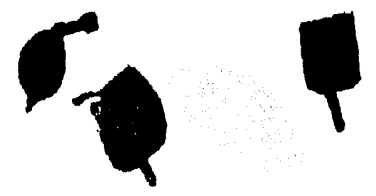

(Right)

the regions. Corresponding regions are then transformed to the proper alignment according to epipolar geometry obtained from the camera calibration procedure. Pixcl-to-pixel correspondence along epipolar lines of an object is accomplished using dymamic programming techniques. The registered pixels are triangulated using the camera transformation matrices. The result is dense range data. Assume that the line of the sight is along the /-axis. The range data thus obtained is a depth map on an irregular grid. We filter the range data and generate the final regular depth map for an object. A 2-D) skeletonization technique is used on the resulting image of the depth map, for grasp analysis.

\section{Camera Calibration}

The accuracy of the 3-1) range data obtained from stereer vision is directly dependent on the accurate moxteling of the camera transformations from 3-I) world coordinates to 2-1)-image pixel coordinates. We are currently using a simple linear model' in which each of the two cameras is modeled by a 3-x-4 perspective transformation matrix, $B$ :

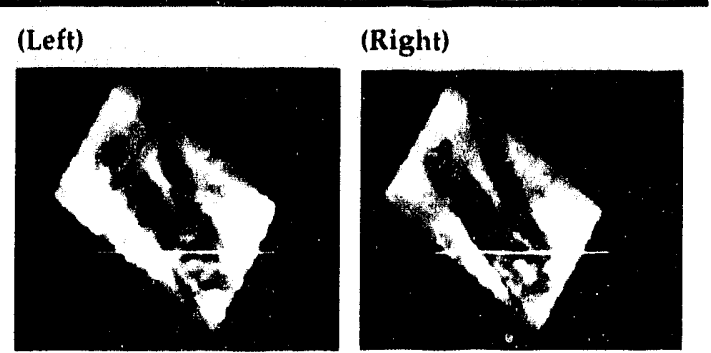

Figure 2. Corresponding epipolar lines (shown in white) for the epipolar-aligned box object.

$$
\begin{aligned}
& \therefore=\mathrm{B} w \\
& \therefore \quad=\left|c_{1}, c_{2}, c_{3}\right|^{\prime} \\
& \therefore \quad=|x, y, z, 1|^{\prime} \\
& \mu_{1}=\frac{c_{1}}{c_{3}}, \quad \mu_{y}=\frac{c_{2}}{c_{3}},
\end{aligned}
$$

where $(x, y, z)$ is a world coordinate and $\left(p_{x}, p_{y}\right)$ is the corresponding image pixel coerdinate. The camera matrices, $\mathbf{B}_{\mathbf{a}}$ and $\mathbf{B}_{\mathbf{b}}$, are solved by the standard least-squares method, using calibration data consisting of world coordinates and their corresponding pixel coordinates. Each sample generates two equations in 12 unknowns for each camera. Therefore, a minimum of six samples is necessiary to solve for the camera matrices. We currently use from 12 to 18 calibration points, by mounting fiducial markers in the robot work cell. The robot (Puma 56(1)) is used to obtain the world coordinates, and centroiding is used to obtain the pixel coordinates. We are currently implementing an automated camera-calibration procedure, in which the robot will move a circular light source around the work cell to specified calibration points, for imaging and centroiding. This will allow for a much more efficient and thorough calibration of the vision system, in which hundreds of calibration points can be used. We expect that this will further reduce the observed positional errors. We are aware of more sophisticated calibration procedures, ${ }^{2}$ in which comern distortion and image acquisition anomalies are modeled. Howerer, for the current application, this additional complexity dees not seem necessary.

\section{Image Segmentation}

A scone withoult objects is taken from each camera an a reference for backgreund removial. Oljects are segmented from the background by first subtracting the reference image from the im- 

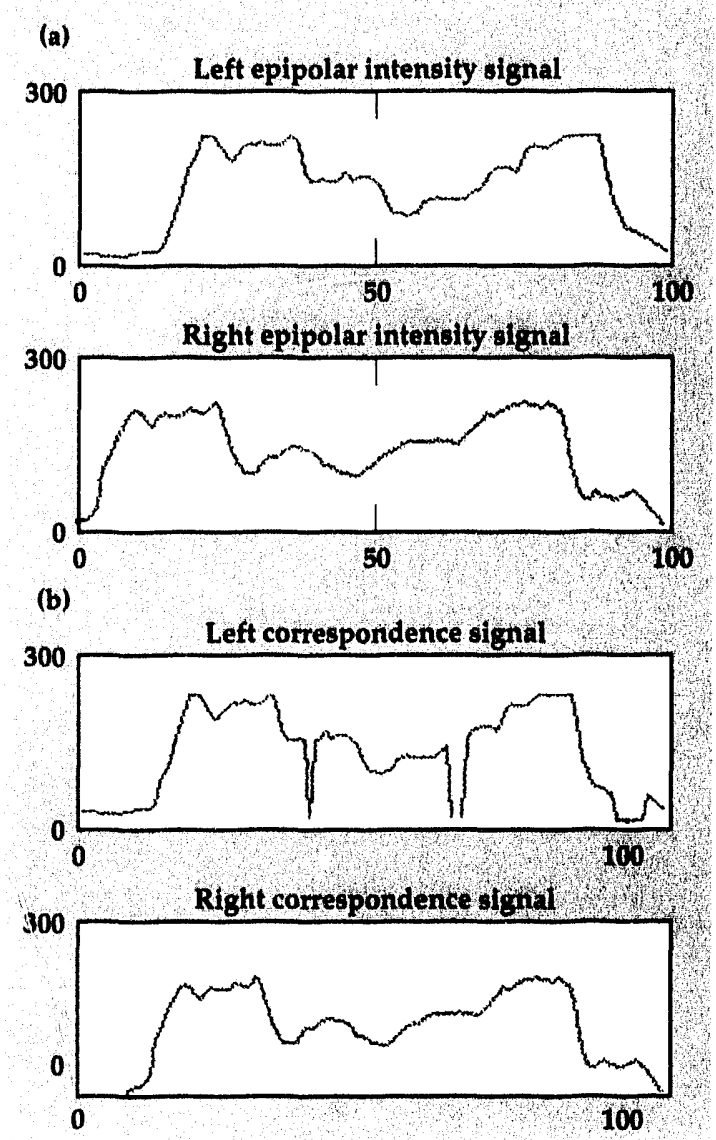

Figure 3. (a) Intensity profiles of epipolar IInes from Fig. 2; (b) realighed correspondence profiles.

age with the objects, and then thresholding the difference image. Size constraints are used to eliminate small, noisy, background-region segments, Regions in one image are matched with regions in the other image, using simple heuristics based on the size and location of the segmented regions. Two stereo images of the scene and resulting corresponded regions are depicted in Fig. 1.

\section{Epipolar Line Registration}

Various dynamic programming techniques have previously been applied to matching edges in stereo images. 3,4 The result is a coarse disparity map for which a complex-surface-reconstruction algorithm is required to generate the final range map. In our approach, pixel-to-pixel registration is done by matching the intensity profiles of two corresponding epipolar lines, using a special dynamic programming technique called dynamic correlation. 'Dynamic correlation is a method that optimally aligns data points, based on a similarity measure, preserving a defined ordering relation. When this technique is applied to stereo registra-

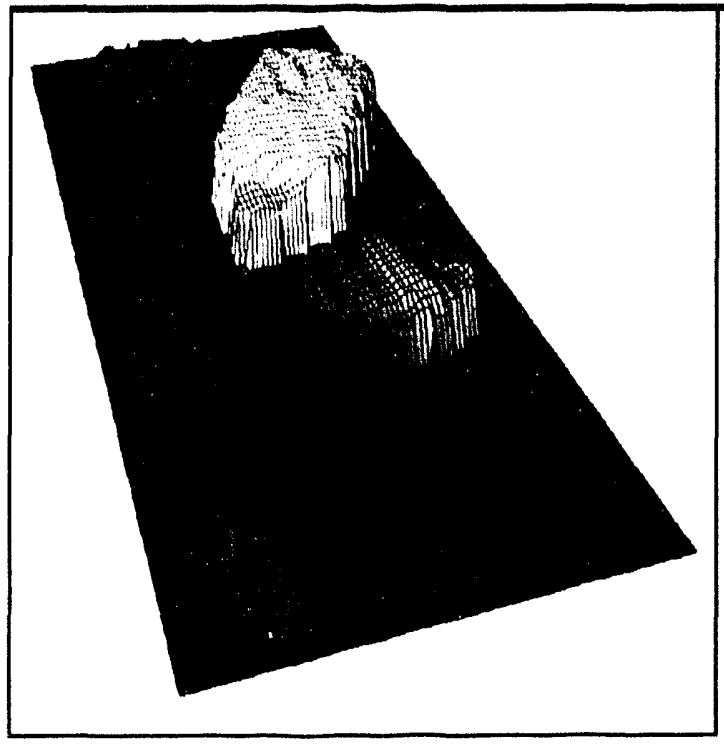

tion, we assume that pixels along epipolar lines have the same left-right relation. This relation can be represented mathematically by a linear ordering relation. ${ }^{6}$ The ordering constraint is generally true for stereo registration, but can be violated. However, a strict linear ordering relation is obeyed by image pixels that pertain to the surface of an opaque object, that is, if pixels $a$ and $b$ are one matching pair and pixels $a^{\prime}$ and $b^{\prime}$ are another matching pair, then if $a$ is to the left of $a^{\prime}$ on one

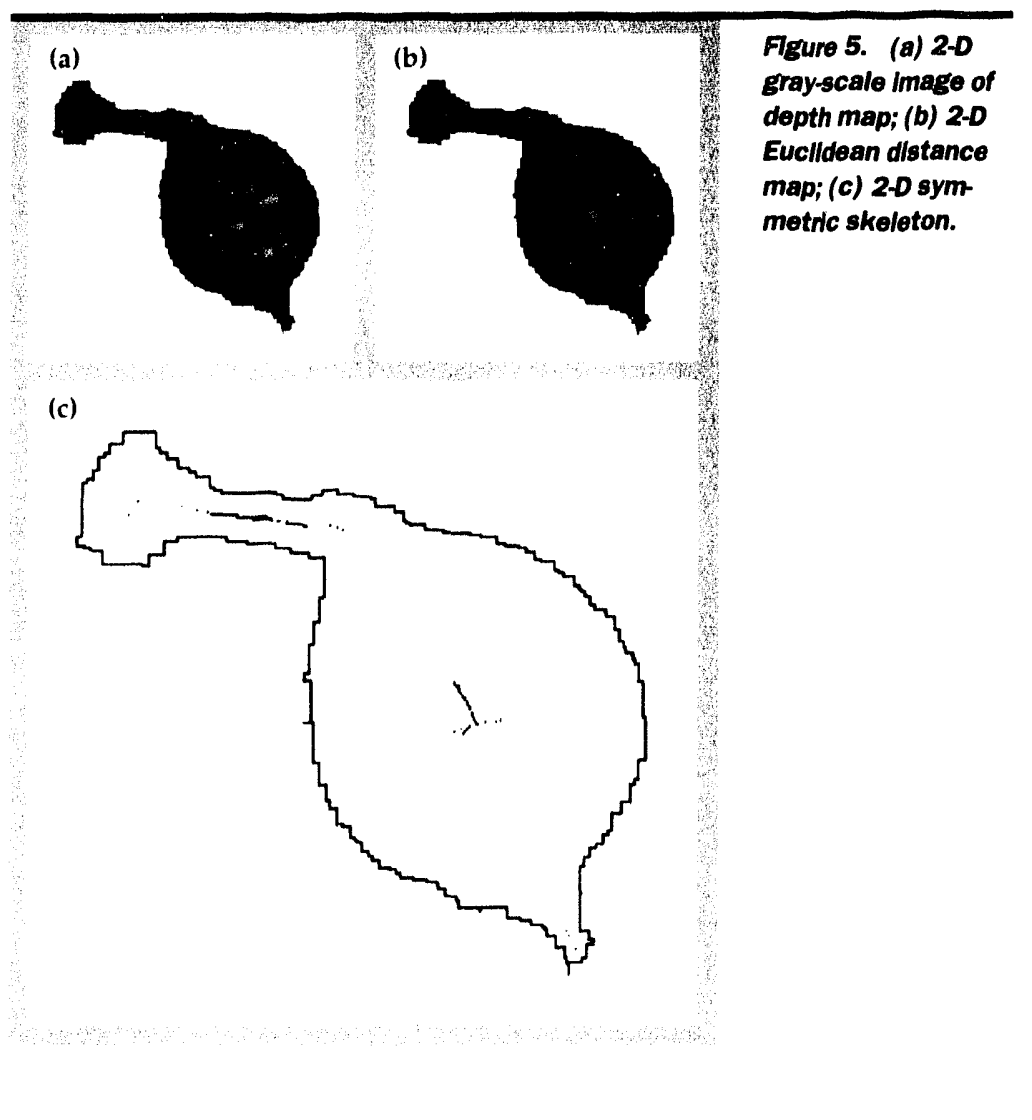

Fgure 4. 3-D recon struction of scene from Fg. 1. 
Fgure 6. Two posstble parallel-grasp orientations.

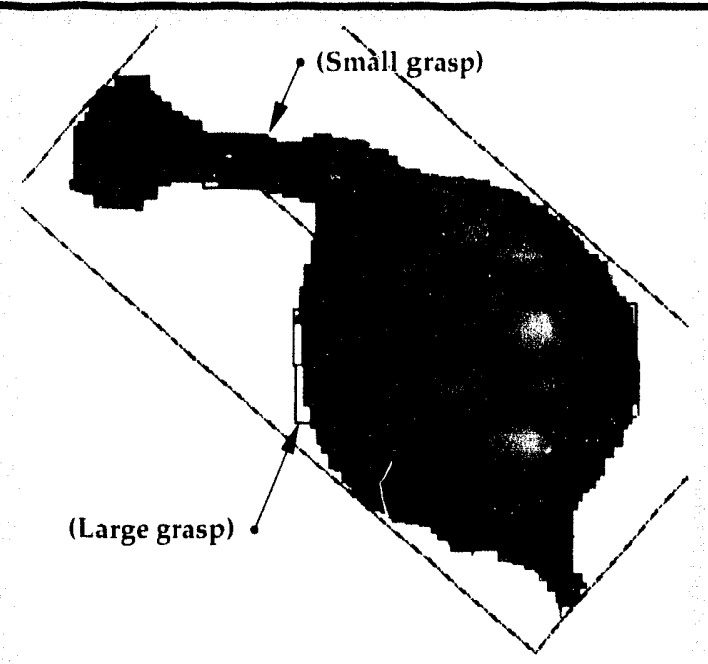

image, then it is necessary for $b$ to be to the left of $b^{\prime}$ on the other image.

Algorithms for pixel-to-pixel registration have to be effective in handling (1) difference in scaling, (2) occlusion, and (3) variation in light reflectance. The deletion (or insertion) operation in dynamic correlation is used to handle both scaling and occlusion. The substitution operation represents a match, but allows variation in brightness. Let $L_{a}$ and $L_{b}$ be two corresponding epipolar lines, and let $a_{i}, i=1,2, \ldots, n$ represent pixels on $L_{a^{\prime}}$ and $b_{j}, j=1,2, \ldots, m$ represent pixels on $\mathrm{L}_{\mathrm{b}}$. The dynamic correlation algorithm calculates the cost of matching $a_{1}, a_{2}, \ldots, a_{i}$ and $b_{1}$, $\mathrm{b}_{2}, \ldots, \mathrm{b}_{\mathrm{i}}$, denoted $\mathrm{C}(\mathrm{i}, \mathrm{j})$ :

$$
C(i, j)=\min \left\{\begin{array}{c}
C(i-1, j-1)+S(i, j) \\
C(i, j-1)+\alpha \\
C(i-1, j)+\alpha
\end{array},\right.
$$

where

$$
S(i, j)=1-\left(\frac{2 R_{u b}(i, j)}{R_{t u t}(i)+R_{b, b}(j)}\right) .
$$

Here, $R_{a b}(i, j)$ is the windowed cross-correlation of $L_{a}$ and $L_{b}$ centered about $a_{i}$ and $b_{i}$ respectively, $R_{a d}(i)\left[R_{b b}(j)\right]$ is the windowed autocorrelation of $L_{a j}\left(L_{b}\right)$ centered about $a_{j}\left(b_{j}\right)$, and $\alpha$ is a fixed cost of deleting an element of $L_{0}$ or $L_{b}$. The normalized substitution cost, $S(i, j)$, varies between 0 and 1 , and the deletion $\operatorname{cost}, \alpha$, is fixed between 0 and 1 . We have achieved good results with $\alpha$. between 0.2 and 0.5. The operation in Eq. 2 defines a minimum cost matrix for $i=1,2, \ldots$, n and $j=1,2, \ldots, m . C(0, j)$ and $C(i, 0)$ are given by $i \alpha$ and $j \alpha$, respectively. The minimum cost alignment can be traced back from
$\mathrm{C}(\mathrm{n}, \mathrm{m})$ to $\mathrm{C}(0,0)$ on the minimum cost matrix. If $C(i, j)$ is derived from $C(i-1, j-1)$, then pixel $a_{i}$ matches $b_{j}$; if $C(i, j)$ is derived from $C(i, j-1)$, then pixel $b_{j}$ on $L_{b}$ does not have a match (a deletion); similarly, if $C(i, j)$ is derived from $C(i-1, j)$, then pixel $a_{i}$ on $L_{a}$ does not have a match.

Two corresponding epipolar lines are highlighted in Fig. 2 for the epipolar-aligned box object from Fig. 1a. The result of pixel-to-pixel registration of these lines is illustrated in Fig. 3. The intensity profiles of the two epipolar lines are shown in Fig. 3a. These two intensity profiles are realigned after using the correlation algorithm. The realigned intensity profiles are shown in Fig. 3b. The matching pixels (substitutions) are aligned. When pixels on one intensity profile do not have a match (deletions), then a blank (shown as a zero value) is filled in on the opposite intensity profile. The occluded portions, i.e., the right-hand side of the box on the right image and the left-hand side of the box on the left image, are successfully deleted by the algorithm. The algorithm handles the slight difference in size (the box is at a larger skew angle to the left camera, therefore it is shown smaller on the left image than on the right image) by deleting four pixels from the right image at scattered locations. The 3-D reconstruction of all four objects from Fig. 1 is shown in Fig. 4.

\section{Grasp Analysis}

Since the objects in this experiment are small, not too tall, and the bag handles are always placed parallel to the table, a simple shape analysis of the 2-D image of the depth map can be used to determine an 'optimum' grasp location with the gripper-oriented parallel table. First, we compute a Euclidean distance map from the 2-D projection of the depth map (see Fig. 5), using the fast raster scan algorithm. ${ }^{7}$ A skeleton is then generated by locating generalized local maxima in the distance map. ${ }^{8}$ Associated with each skeleton point is a vector pointing to the closest image point not contained in the object region. This orientation information is used to identify symmetric skeleton points (see Fig. 5) with respect to the object region boundary. Using information about the current available grippers, a grasp feature vector is then computed for each symmetric skeleton point, consisting of positional information, a grasp size, a parallelboundary deviation measurement, and the distance from the object region centroid. Finally, a specified optimality criterion is used to 
choose an optimal grasp. Our current criterion consists of first only considering grasps within the range of the current grippers and with parallel deviations less than a specified maximum. Of those, the grasp that minimizes a weighted average of parallel deviation and distance from centroid is chosen as the optimal grasp. In practice, we often divide the current grippers into two groups, (1) small grippers and (2) large grippers. We then find a grasp for each group. For the current objects, this often gives a large grasp about the center of mass and a small grasp about the bag handle, as shown in Fig. 6.

\section{Future Work}

Preliminary results of applying stereo vision and shape analysis to robot autonomous grasping of unknown objects show that stereo vision can provide fast and reliable range information. So far, we have used a relatively simple approach for grasp planning. We expect to deal with more complex objects as the waste sorting project progresses. We are also interested in the proper mating of object geometry and manipulator geometry, and plan to use special hardware such as transputers to speed up the process for real-time applications.

\section{Acknowledgements}

The authors would like to thank Maynard Holliday and the Advanced Processing Technology Program of Lawrence Livermore National Laboratory for their support and for the use of the robotic facilities in the Interactive Controls Laboratory.

1. D.H. Ballard and C.M. Brown, Computer Vision, Prentice-Hall, (Englewood Cliffs, New Jersey) 1982.

2. R.Y. Tsai, IEEE I. Robotics and Automntion RA-3, 323 (1987).

3. Y. Ohta and T. Kanade, IEEE Trans. Pattern Anal. and Mach. Intell. PAMI-7, 139 (1985).

4. S.A. Lloyd, E.R. Haddow, and J.F. Boyce, Computer Vision, Graphics, and Image Processing 39, 202 (1987).

5. S.Y. Lu, "A String-to-String Correlation Algorithm for Image Skeletonization," Proc. 6th litt. Joint Conf. Pattem Recognition (Munich, Germany), 178 (October 1982).

6. A.V. Aho, and J.D. Ullman, The Theory of Parsing, Translation, and Compiling, Prentice-Hall (Englewood Cliffs, New Jersey), 1972.

7. F. Leymarie and M.D. Levine, CVGIP: lmage Understanding 55, 84 (1992).

8. U. Montanari, J. Assoc. Computing Machinery 15, $600(1968)$. 


\title{
Image-Restoration and Image-Recovery Algorithms
}

\author{
Dennis M. Goodman \\ Laser Engineering Division \\ Electronics Engineering
}

We have written computer codes for solving various image-restoration and image-recovery problems. These codes are based on a variant of the conjugate gradient algorithm that permits the imposition of constraints. Although the codes are essentially spatial-domain methods, most of the computation is done in the frequency domain. The result is that the flexibility of spatialdomain methods is preserved, but computation time is closer to that of conventional frequencydomain methods.

\section{Introduction}

A crucial tradeoff in applying image-processing algorithms to restoring a blurred image or recovering an image from data is accuracy vs time. Standard algorithms are non-iterative and operate in the frequency domain. Suppose the image is an array of $\mathrm{N}-\mathrm{x}-\mathrm{N}$ pixels. The number of floating point operations (FLOPS) required by these algorithms is typically of order $N 2 \log N$, the same order required for computing an $\mathrm{N}-\mathrm{x}-\mathrm{N}$, two-dimensional, fast Fourier transform. Consequently, frequency-domain methods are reasonably fast; unfortunately, they are not flexible enough to impose non-negativity constraints, to handle nonlinear problems, or to deal with 'ringing' effects that occur when the blurred image is not zero at its boundaries.

The solution is to use spatial-domain methods, but the price paid in computer time is very high. Because direct inversion methods involve the storage and inversion of an $\mathrm{N}^{2}-x-\mathrm{N}^{2}$ matrix, these methods are impractical for all but very small images. Instead, iterative methods are used. A typical iterative method computes one convolution and one correlation per iteration. If these are implemented in the spatial domain, each requires on the order of $N^{4}$ FLOPS, so the total number of FLOPS required by an iterative method is on the order of $\mathrm{MN}^{4}$ where $\mathrm{M}$ is the number of iterations. For small $\mathrm{M}$, iterative methods are much faster than direct inversion methods, but are still much slower than frequency-domain methods. In fact, they are not practical for images larger than $128 \times 128$ pixels.
We have developed a new method that is basically a spatial-domain technique, but implements the iterations in the frequency domain. This reduces the cost per iteration to the order of MN2 $\log N$ FLOPS. Our particular iterative technique is also new. It is based on the conjugate gradient algorithm and uses a 'bending' line search strategy, a special implementation of the active set strategy' and the Hestenes-Stiefel formula.

\section{Progress}

In FY-91, we applied this algorithm to the standard, linear, least-squares image-restoration problem. We were able to demonstrate that the imposition of positivity constraints and the ability to properly handle boundary effects greatly enhanced image quality. This year, we performed Monte-Carlo experiments on small data problems, which demonstrated that the estimates obtained with our technique ${ }^{1}$ were at least as good as those obtained with more conventional methods, such as constrained regularization and maximum entropy. As noted above, the conventional spatialdomain methods are too slow to apply to large data problems.

Many image-restoration and image-recovery problems are inherently nonlinear. For example, the algorithm we developed for the standard, linear, least-squares restoration problem is inappropriate when imaging at very low light levels. This is because the quantum nature of light must be accounted for, and the noise must be modeled as Poisson, rather than Gaussian. The result 
Figure 1. Blurred Image with Polsson nolse.

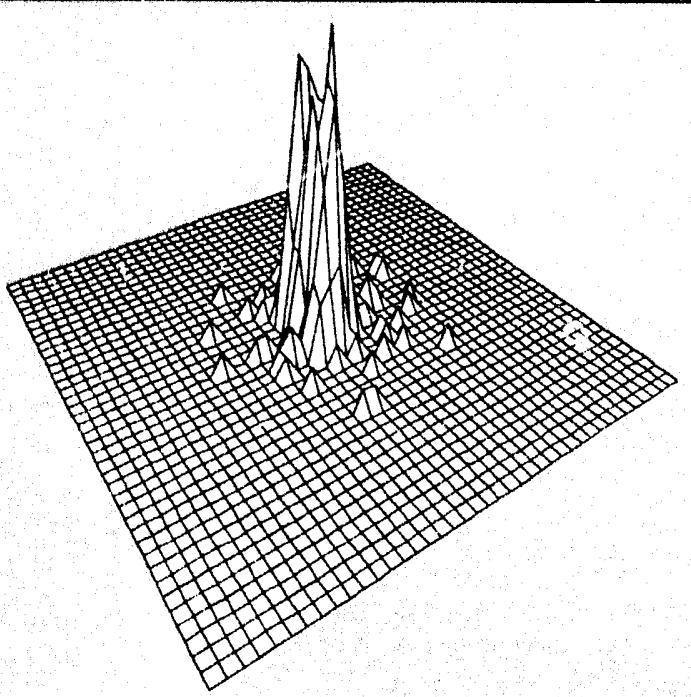

Figure 2. Estimate obtained using a least-squares cittent on.

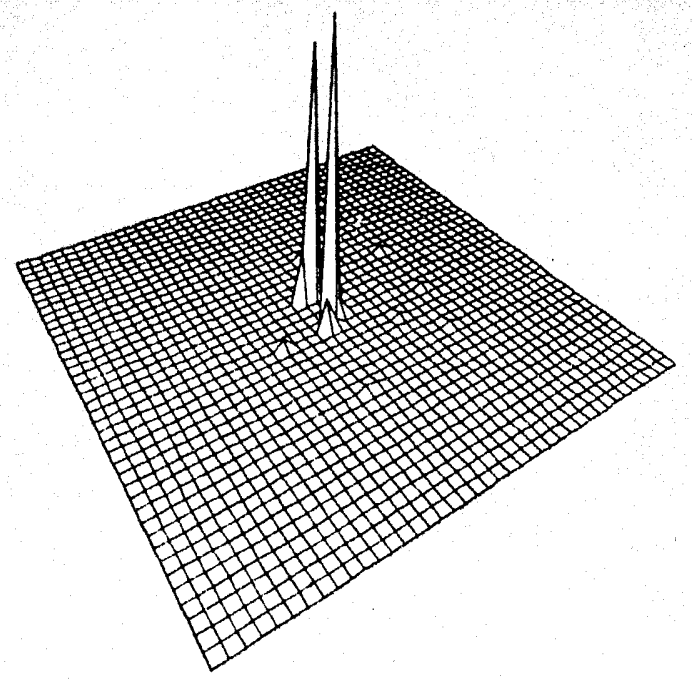

Figure 3. Estimate ohtained by maximiz. Ing the Poisson Ilkell. hood function.

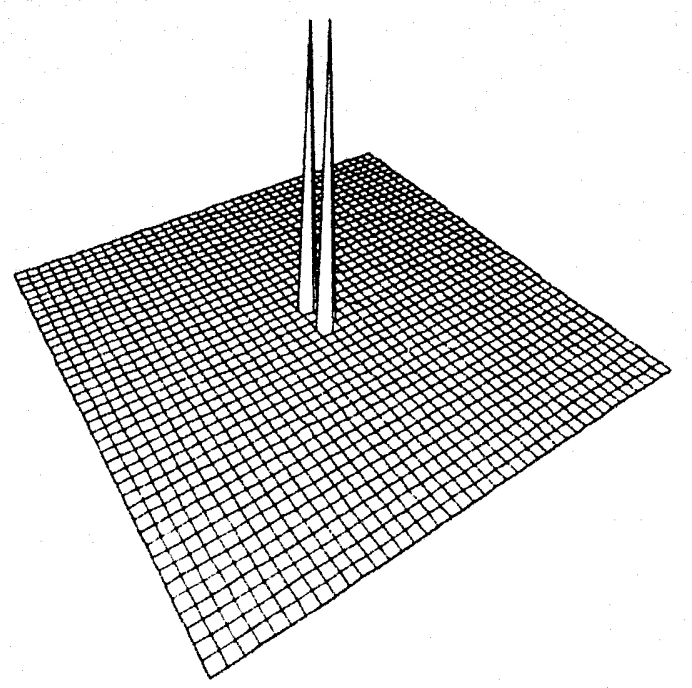

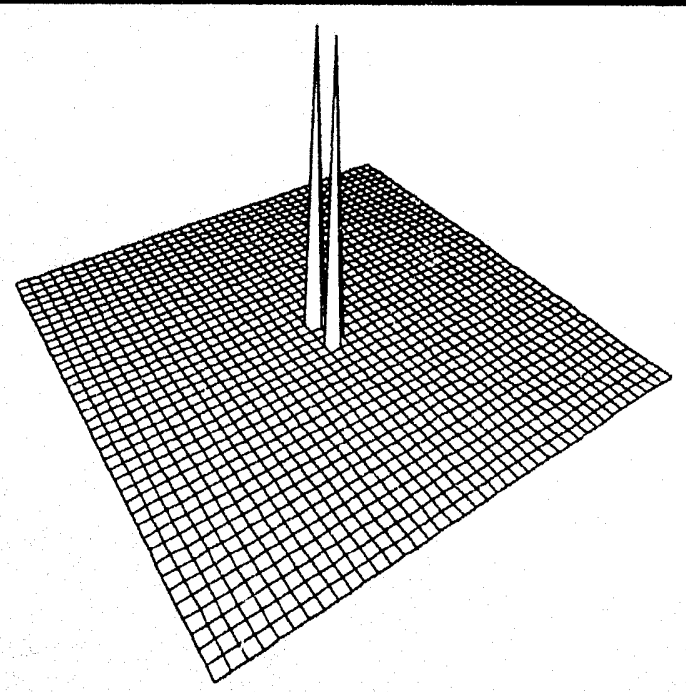

Figure 4. True noise-free image.

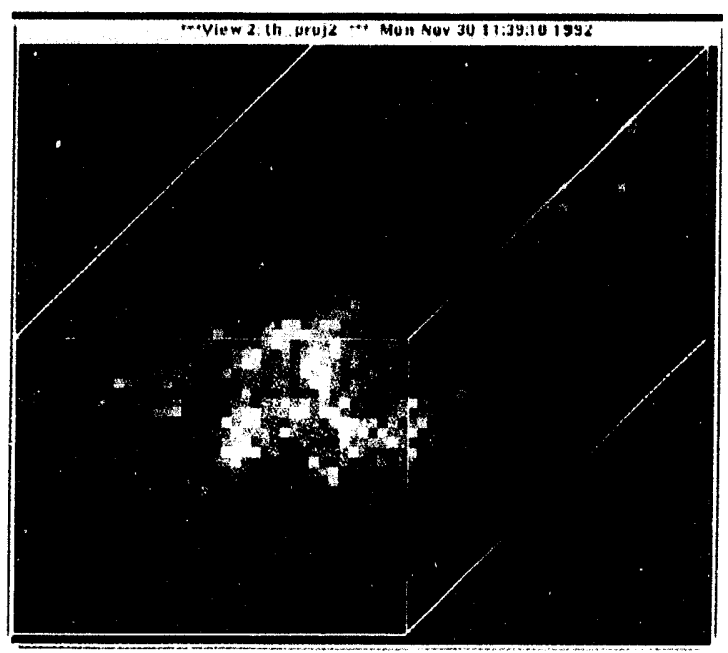

Figure 5. Reconstructed, three-dimensional, unit crystal cell for the protein thaumatin.

is a highly nonlinear likelihood function that must be maximized. In FY'-92, we extended our algorithm to perınit minimizing or maximizing general nonlinear functions. ${ }^{2}$ Figure 1 is a simulation of the result of imaging two closely spaced point sources through a circular aperture at a low light level. The result of deblurring using the least-squares criterion is shown in Fig. 2; the result of deblurring by maximizing the likelihood function for Poisson noise is shown in Fig. 3. The noise-free image is shown in Fig. 4. The estimate obtained by using the proper noise model is clearly superior.

We have also applied our algorithm to several other nonlinear imaging problems. These include speckle interferometry, holography, and crystallography. A crystallographic example is show'n in 
Fig. 5. This image is a three-dimensional reconstruction of the protein thaumatin. The reconstruction is obtained using the Eden algorithm, which uses our algorithm as an inner iteration to repeatedly solve a non-negative least-squares problem consisting of 28,000 equations in 36,000 unknowns. The solution shows excellent clustering of the residual scatterers, since the reconstructed information occupied only $16 \%$ of the available grid positions.

\section{Future Work}

We plan to continue work on the crystallography problem in FY-93.
1. D.M. Goodman, "Deconvolution for Positive Signals," Funtame'ntals of Discrete-Time Systems, M. Jamshidi (Ed.), 1993.

2. D.M. Goodman, E.M. Johansson, and T.W. Lawrence, "On Applying the Conjugate Gradient Algorithm to Image Processing Problems," Multivariate Analysis: Future Diretions, C.R. Rao (Ed.), North Holland, 1993.

3. D.M. Goodman, T.W. Lawrence, E.M. Johansson, and J.P. Fitch, "Bispectral Speckle Interferometry To Reconstruct Extended Objects from Turbulence Degraded Telescope Images," Hanthook of Statistics, Vol. 10: Signal Processing and its Applications, N.K. Bose and C.R. Rao (Eds.), North Holland, 1993. 


\section{View: A Signal and \\ ImageProcessing System}

\author{
James M. Brase, \\ Sean K. Lehman, and \\ Melvin G. Wieting \\ Laser Engineering Division \\ Electronics Engineering
}

\author{
Joseph P. Phillips and \\ Hanna Szoke \\ Scientific Softuare Division \\ Computation Directorate
}

View is an interactive signal- and image-processing environment for UNIX workstations with the X11 window system. View provides tools for image enhancement and general signal analysis. The system is used in programs at Lawrence Livermore National Laboratory for experimental data analysis and has been distributed to university, government, and industrial users.

In FY-92, we developed a capabili'y to handle very large signal databases containing large numbers of signals or images with accompanying descriptive information; we continued to enhance the base View language, algorithms, and tools, and we demonstrated a prototype tool for distributed signal processing on a workstation network.

\section{Introduction}

A project to develop View, ${ }^{1}$ an interactive signal- and image-processing environment for UNIX workstations with the X11 window system, was started at Lawrence Livermore National Laboratory (LLNL) in 1986. Early work focused on tools for image enhancement and analysis for nondestructive testing applications. View has been used extensively for image analysis for radiography and computed tomography as well as signal processing for ultrasonic imaging. Our development has continued with applications centered on radar imaging, remote sensing applications, and highresolution astronomical imaging, including speckle interferometry and adaptive optics.

View provides most of the tools commonly required for signal and image analysis. Interactive capabilities include color map manipulation, lineout and region extraction, data value display, and image annotation. View's command interpreter provides a general purpose signal-manipulation language with looping and conditional constructs. The signal- and image-processing capabilities include spectral analysis, smoothing and sharpening filters, and adaptive noise-reduction techniques.

View continues to be used in the applications described above as well as in others at LLNL. It is the principal system used for reconstruction of $x$ ray holograms and microscopy and as a diag-

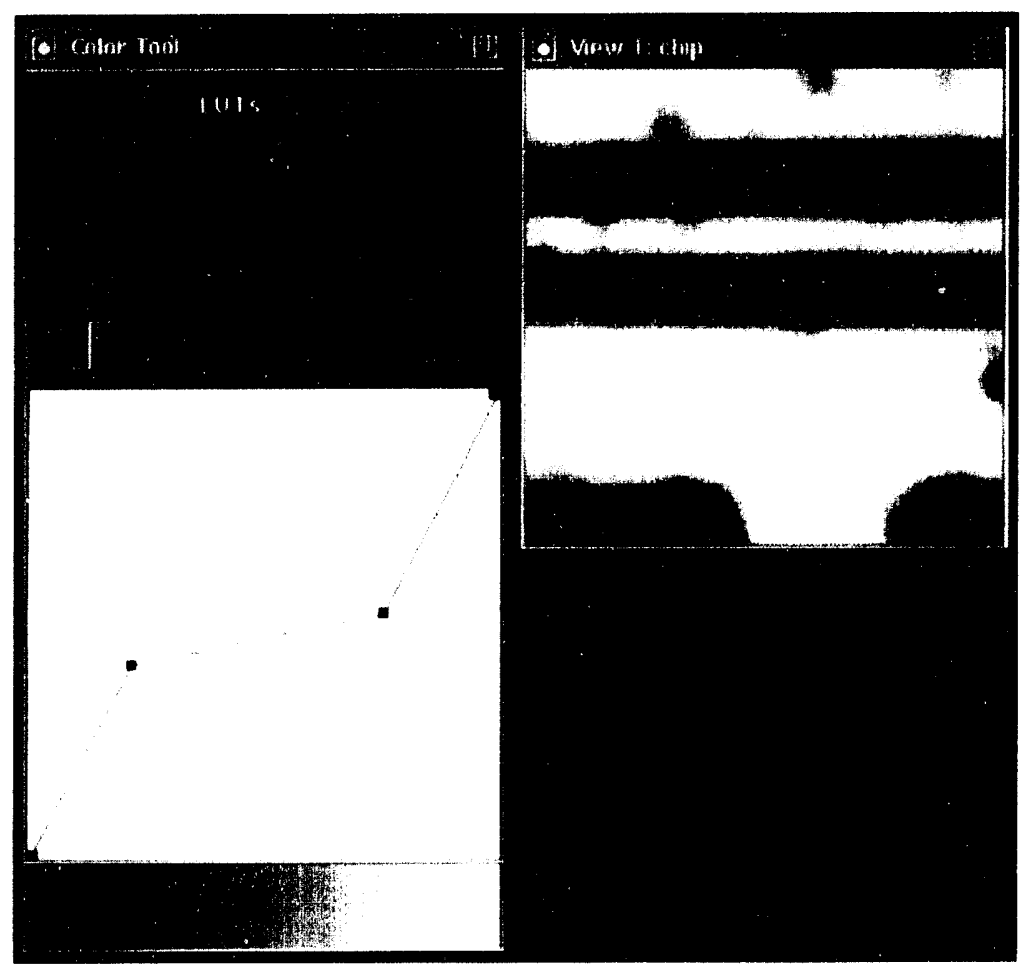

Figure 1. Image processing results from our new tool for interactive color map ma nipulation, allowing piecewise linear color maps. 


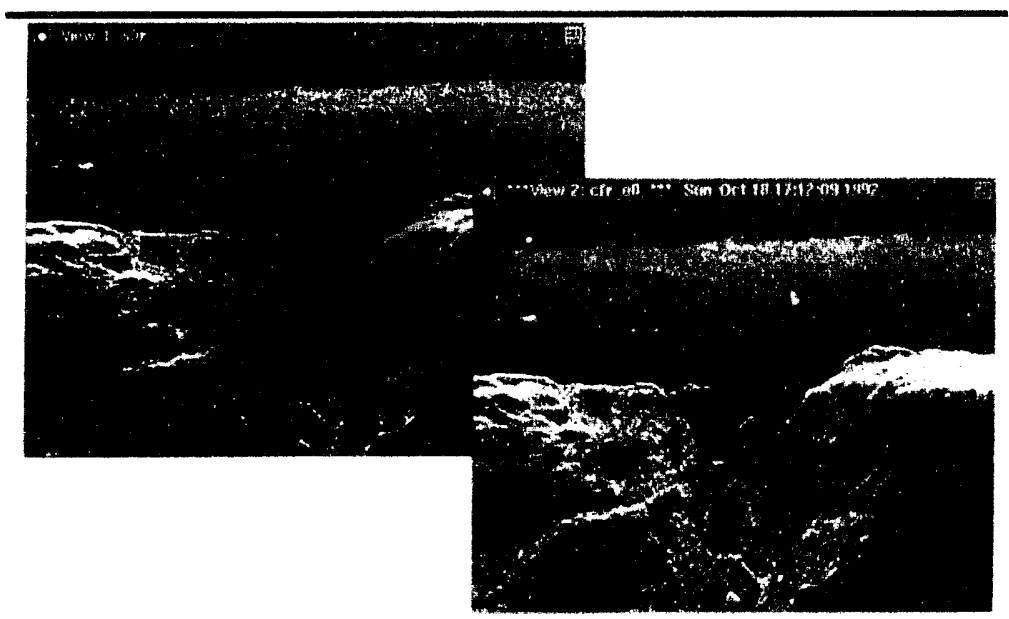

Figure 2. Image sharpening from a new two-channel technique added to View: (a) the original image; (b) recombined component images.

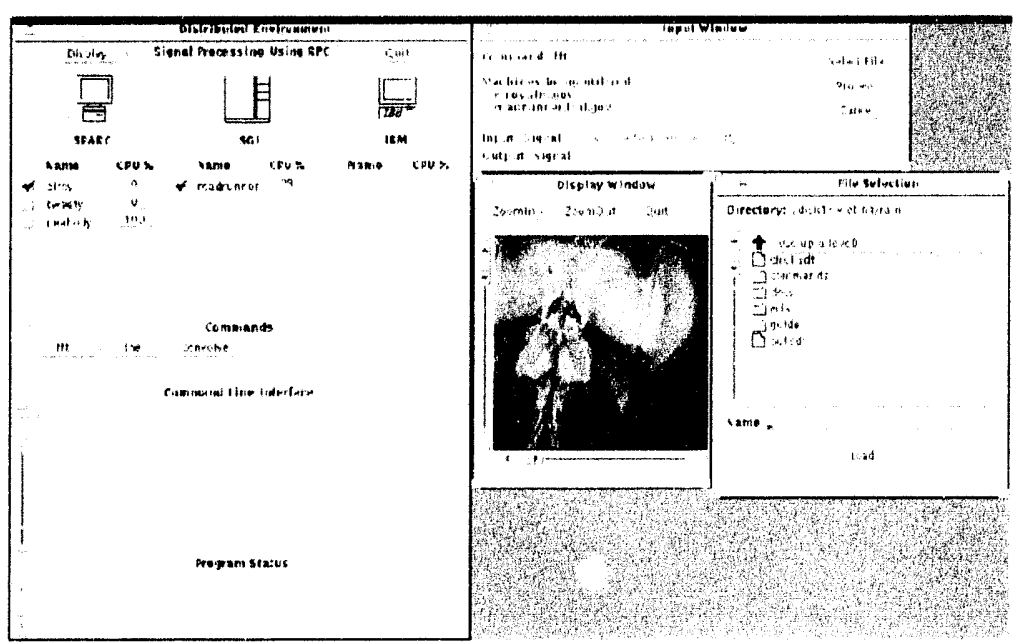

Figure 3. Graphical user interface from a prototype system for distributed signal processing. The user can assign parts of computations to dif. ferent workstations on the network. nostic tool for magnetic fusion experiments. ()utside I.L.NI, View has been used for a wide variety of applications, including coding research, industrial tomography, and silicon wafer inspection. It has also served as a $t(x)$ to teach basic signal and image processing at several universities.

\section{Progress}

Our development work on View in the past vear has focused on three main areas: (1) the development of tools for handling harge signal databases; (2) general enhancement of the wailable signal-processing algorithms and tools; and (3) the demomstration of a protenlype distributedsignal-prexesing stestem.

The sigmal databuse in the heart of the View sistem. It comprises all the data homenn to the sistem aleng with asereiated information on its oreamisation and characteristics. The original signal database had a flat structure, it wasesentialle alist of aigmalu that could be accessed. This strkfure interest the organisation of analysis work on the user, either by using oonsistently meaningtul names or simply by memory.

$A$ better design is a hierarchy of signal groups or directories, similar to the way that most computer file systems are organized. With this structure, sigmals that are logically connected can be grouped together in the database structure. Signal names can be kept shorter, but the directory structure can still maintain a large amount of contextual information.

Our implementation of a hierarchical signal database is based on the UNIX file system. The database is a tree structure of signal directories that the user can more through and manipulate using a set of View commands. The commands have the same names as those in UNIX: mkdir to create a new subdirectory; cd to change the working directory; and pwd to print the current working directory. All other View operations can be executed normally and use the current working directory for signal storage, unless a specific path is given.

We have alse developect a new capability to link the signal database to the existing Unix file system. If View is started with a "-u" option, a new signal database will be created that matches the UNIX directory structure starting at the current directory. All View data files in those directories will show' up in the View internal-signal database, automatically. As the user moves through the signal database, the working UNIX directory changestomatch it.

This linkage can greatly ease working with large amounts of data created $1 \%$ an external program and stroed in a hierarchical file structure. We can immediately match the View database structure to the application.

Our second focus, continued develepment of new toxls and algorithms for signal and image processing, has resulted in a new tool for interactively manipulating color maps (Fig. 1). This development allows piecewise linear mapping from data values to colors (shown in shades of gray), a valuable capability for images with multiple histogram peaks. The new color teol also allows the design of completely new color tables through interactive specitication of red, green, and blue colormaps.

Algorithm develepment is driven largely by the applications of View. Some of the new terhnicpues aded recently indudeadaptivesmenthing filters for additise and multiplicative no rise, improsed spectro gram estimation for nomstationary signals, and basic capabilition for waded andysis. A new twerchannel texhnigue for nonlinear image sharpening is shown 
in Fig. 2. On the left is the original image. Weseparate it into low- and high-frequency components and apply a nonlinear sharpening operation to each component.? The component images are then recombined to get the result on the right. This algorithm has proven useful in enhancing synthetic aperture radar imagery.

Our third development area in FY-92 was the demonstration of a prototype tool for distributed signal processing. The system allows operations to be distributed over a network of UNIX workstations. An interactive graphical user interface(Fig. 3) allows the user control over which machines run each command. Signal communication is through the network file system. These capabilities will form the base for future enhancements of View.

\section{Future Work}

Network-based distributed workstations will continue to be the foundation for our efforts in high-performance signal and image processing. View will be further developed to support both databases and computations that span the network. We are currently redesigning the signalprocessing language and its interpreter to support these capabilities. We also plan continued development of user interface enhancements to conform to emerging standards in graphical user interfaces. Algorithm development will continue to be driven by ongoing applications.

1. J. Brase, V. Miller, M. Wieting, H. Szoke, and J. Phillips, The Vie'r' Signonl and linage' Processing System, Lawrence Livermore National Laboratory, Livermore, California, UCID-21368 (1988).

2. S.K. Mitra, H. Li, I. Lin, and T. Yu, "A New Class of Nonlinear Filters for Image Enhancement," Proc. Int. Conf. Aconstics, Speech, and Signal Processing (Toronto, Canada), (1992). 


\title{
VISION: An Object-Oriented Environment for Computer Vision and Pattem Recognition
}

\author{
Jose E. Hemandez and \\ Michael R. Buhl \\ Engineering Research Division \\ Electronics Engineering
}

VISION is a flexible and extensible object-oriented programming environment for prototyping solutions to problems requiring computer vision and pattern recognition techniques. VISION integrates signal/image processing, statistical pattern recognition, neural networks, low- and mid-level computer vision, and graphics into a cohesive framework useful for a wide variety of applications at Lawrence Livermore National Laboratory.

\section{Introduction}

During the past two years, we have been developing an object-oriented programming environment known as VISION, for computer vision and pattern recognition. VISION is a hybrid system consisting of C, Lisp/CLOS,1,2,3 and some FORTRAN code. CLOS, the Common Lisp Object System, defines the new standard for object-oriented programming in the Common Lisp language.

The VISION system was developed with several goals in mind: (1) to provide a technology base at Lawrence Livermore National Laboratory (LLNL) in computer vision and pattern recognition; (2) to provide support to programs at LLNL requiring this technology; and (3) to provide a software package capable of being extended and customized directly by the end users.

During FY-91, most of the object-oriented framework was developed, including basic classes of data structures for signal/image processing, midlevel two-dimensional (2-D) computer vision, and unsupervised and supervised learning algorithms including several neural networks, 4,5 Some of the capabilities in VISION were applied to several projects sponsored by LLNL's Earth Sciences Department. Also, VISION was used as a development environment for the temperature-evaluated mine position survey (TEMPS) project for locating buried mines.' This preliminary work resulted in a 1.5-millior-dollar project currently funded by the
Defense Advanced Research Projects Agency, and a possible licensing agreement with a private company.

During FY-92, VISION was used as the development environment for a research project in stereo vision and grasp planning for robotics. This effort resulted in a demonstration system currently being used at LLNL's Interactive Controls Laboratory managed by the Advanced Processing Technology Program. Some of the capabilities in VISION have also been integrated into LLNL's Seismic Expert System,7 sponsored by the Treaty Verification Program.

In FY-93, VISION will be used to prototype pattern recognition algorithms for LLNL's INSENS project, Broken Heart Valve project, and wake detection project, and for developing more advanced capabilities in computer vision for robotics.

\section{Overview of VISION}

VISION consists of two major parts: the programming environment, and the computer vision and pattern recognition capabilities. The programming environment is primarily provided by the Common Lisp environment itself. Some of its features are listed below.

(1) Interactive programming: eliminates the need to write a command-driven user interface and encourages incremental development;

(2) Run-time linking: C, FORTRAN, and compiled Lisp code can be loaded and linked dynamically at run-time; 
Figure 1. An inter active class browser, useful for under standing the VISION class system.

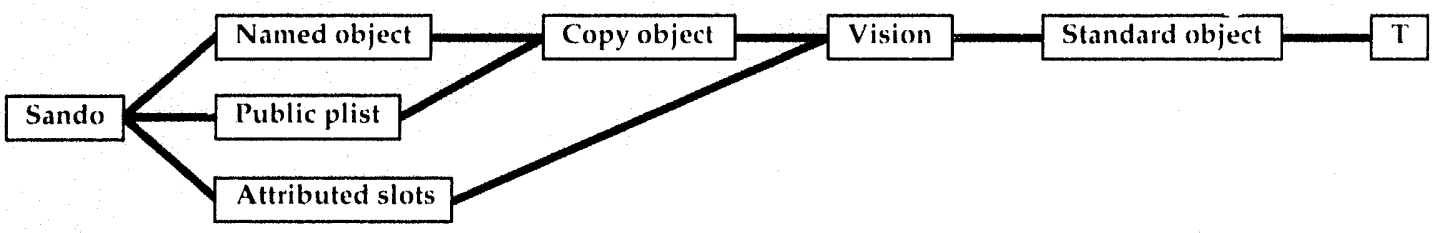

(3) Automatic memory management: Lisp handles the allocation and deallocation of memorry, hence code can be developed faster;

(4) Loosely typed language: since there is no need to declare data types, algorithms can be prototyped faster;

(5) Functional programming: we have the ability to dynamically define functions to be passed as arguments to other functions, which is essential to our framework for pattern recognition;

(6) Object-oriented progranming; Lisp supports the object-oriented programming paradigm, which is essential for our extensible framework, through CLOS;

(7) Emacs interface: expressions, regions, and buffers within Emacs can be submitted to the Lisp interpreter directly, which improves productivity;

(8) Artificial intelligence (AI) software: Lisp software is available in the public domain for supporting many of the $\mathrm{Al}$ paradigms for high-level reasoning; and

(9) Class browser: an interactive class browser based on C.ARNET is available for browsing the VISION class system (see Fig. 1). (GARNET is a Lisp-based graphical user interface environment developed at Carnegie Mellon University:

The computer vision and pattern recognition capabilities in VISION consist of an integrated set of data structures and algorithms within an object-oriented framework for (1) representing, processing, and segmenting one-dimensional (1-D), 2-D, or three-dimensional (3-D) data; (2) calculating and evaluating features for statistical pattern recognition; and (3) several paradigms for object recognition and classification including neural networks and an Assumption Truth Maintenance System." In summary, VISION is an extension to the Common Lisp environment to make it more useful for signal/ image processing, pattern recognition, and com-

\section{Progress}

In FY-92, there were a substantial number of improvenents and developments in the areas of computer vision and pattem recognition made to the FY-91 VISION relcase.

\section{Collection Objects}

The class Collection-Object is one of the most fundamental building blocks in VISION. This class unifies many of the data structures in VISION for storing collections of other data structures. Perhaps the most important aspect of this class is that it prorvides many methods for implementing generic higher-order functions (GHOF)." These are generic functions that accept other functions as arguments to puter vision. (a)

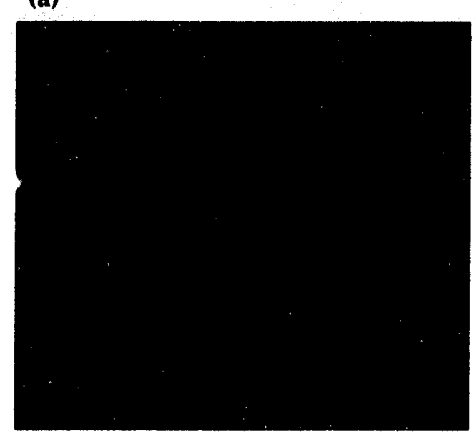

(b)

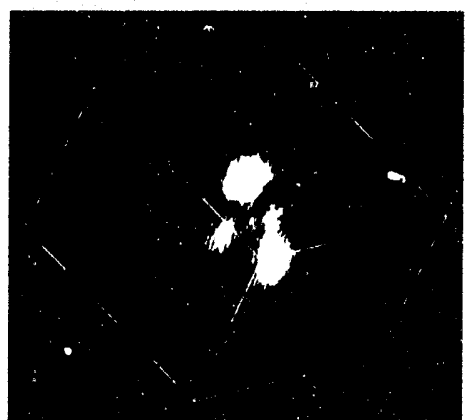

(c)

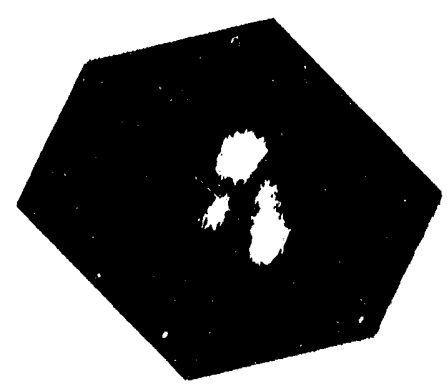

Figure 2. (a) 3-D data representing a cell, (b) thresholded data. Each voxel in the volume is assigned to one of three intensity bins represented by the three different gray levels. (c) Regions identified within the voxels in the third intensity bin. Three chromosomes were found. 
(a)

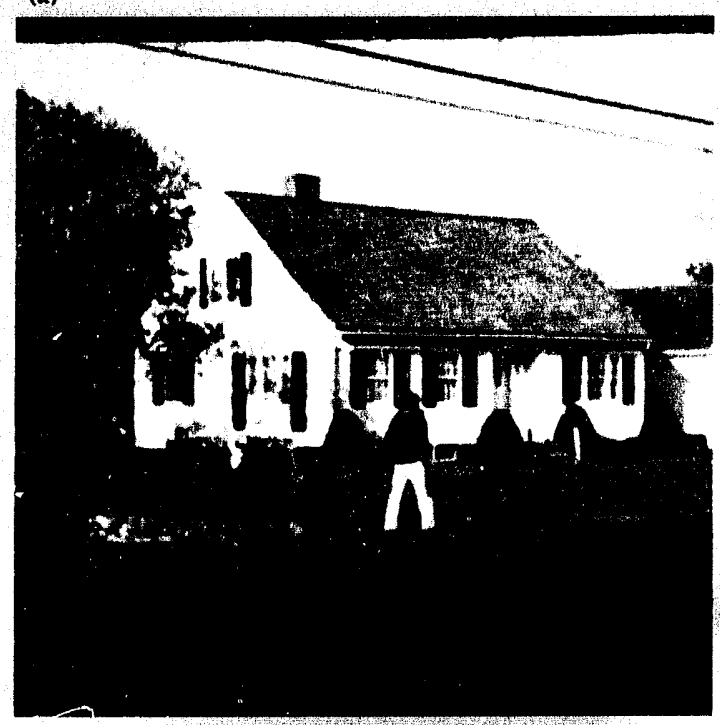

be applied to the individual objects in the collection. Generic functions ${ }^{2}{ }^{3}$ are functions for which methods can be defined to provide the appropriate functionality for different classes of objects. GHOF provide a powerful mechanism for solving problems without the explicit use of recursion or iteration. Furthermore, they hide the internal representation of the collection object, since the iteration process is hidden. For example, consider the GHOF gcount-if, which counts the number of objects in the collection that satisfy a predicate (test). In the context of computer vision, wecould use this function to count all the round objects in a segmented image,

(gcount-if \#'roundp segments) $\Rightarrow 52$

In general, algorithms can be prototyped faster, since we only need to develop primitives that deal with the individual objects in the collection. We can then use lambda expressions to combine these primitives and form more complex expressions that can be applied to the individual objects in the collection. Lambda expressions' are anonymous functions typically defined to be passed as arguments to other functions. For example, we could use a lambda expression to count all the signals from a collection of time series that have a positive mean,

$$
\begin{aligned}
& \text { (gcount-if \#'(lambda }(x) \\
& \quad \text { (plusp (mean } x)) \text { ) signals) } \\
& \Rightarrow 11
\end{aligned}
$$

Some examples of subclasies of the class Collextion-Object are: Signals, for storing a collection of $1-D$ waveforms; Monochrome-Images, for storing a collection of 2-D images; and Segmented-Image, for storing a collection of region segments in an image.

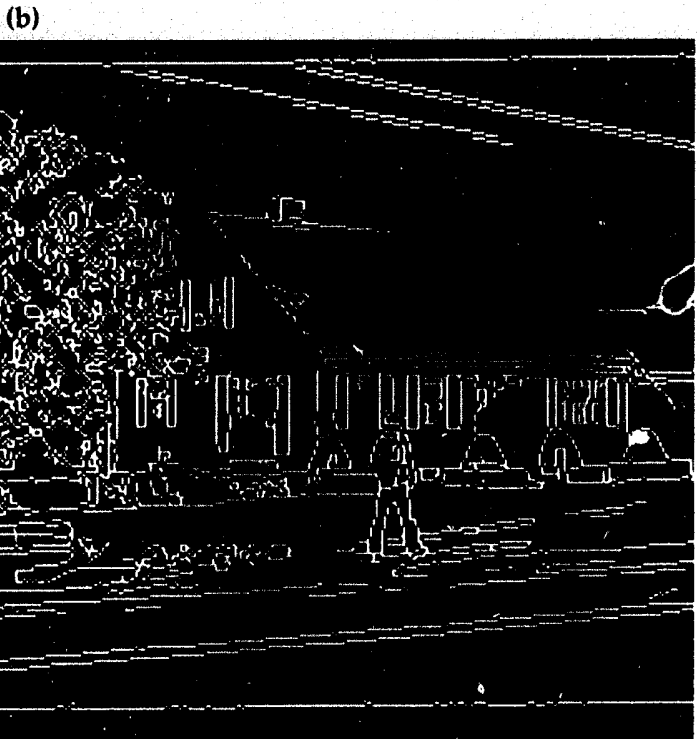

Figure 3. Example of the use of an auto matic thresholding algorithm on the gradient of an image. Pictured are (a) a house scene and (b) edges of the image.

\section{Volume Segmentation}

An object-oriented framework for 3-D data segmentation has been developed. The new capabilities are very similar to the computer-vision capabilities that were developed last year for 2-D data. In fact, the original, 2-D, computer-vision framework was redoneso that it could beextended to any $\mathrm{N}$-dimensional space. Due to our limited resources, there are no plans at this point to develop 3-D graphics capabilities in VISION. However, an interfince was developed to write the different classes of 3-D data objects in VISION to disk in SUNVISION format for data visualization. SUNVISION is a $3-D$, interactive visualization program available for the SUN workstations. LLNL currently has a site license for SUNVISION. An example of a segmented volume showing the nucleus and chromosomes of a cell is shown in Fig. 2.

In summary, the new 3-D capabilities include:

(1) multi-level thresholding of $3-D$ data,

(2) representation and processing capabilities for any arbitrary set of voxels in the volume (connected or non-comnected),

(3) 3-D grouping algorithm for identifying 3-D 'regions' in the volume,

(4) basic-shape-analysis capabilities, and

(5) intertace to SUNVISION for 3-D data visualization.

\section{Automatic Thresholding}

Several algorithms were developed for automatic thresholding of data. I Some of these algorithms are useful for separating background from non-back- 


Figure 4. (a) An
Image from an Infra-
red sensor showing
several objects,
(b) three sections in
the image, classified
as burled mines
using a neural net-
work.

(a)

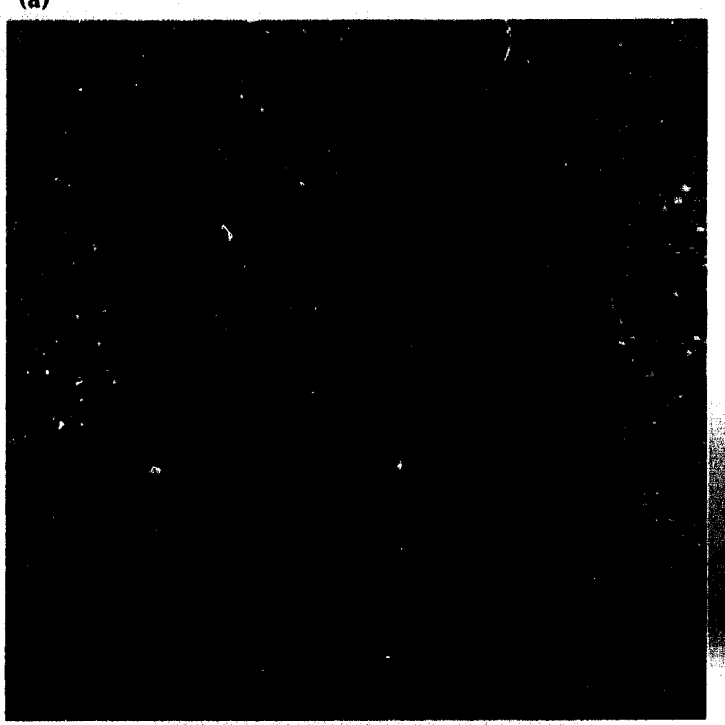

ground pixels. They are also very useful for autematic thresholdting of gradient images for edge detection (sce. Fig. 3).

A multi-thresholdting algorithm was also developect, basert on a K-means ingerithm that clusters the data values directly from a histegram and therefore it is very fast. The algorithm also fentures the ability to find the best number of threshold values based on the ratio of the scatter-matrices. 12 This algerithm is currently being evaluated for segmenting $x$ mys of suitcasss taken at airports, for detecting explesives.

\section{Feature-based Object Recognition}

Many algorithms were developed for extracting features for object recognition. Some of them are listertbolow.

(1) I fistogram features: also known as first-order fentures, uset tecextract features from the probability density function of the data;

(2) Central moments: conbeused toextract shape information and are inviariant to thansiation;

(3) Ilu moments: similar to the central moments, but they arealse inviariant to rotation; and

(4) Texture features: also known as second-order fertures, used torextract texture fentures from an inlige.

Many other algerithms are also avilable for extracting information that could be used as features for object recognition. These features conn be used as inpul tor rule-based system or to a neural notwork forobject recognition. (b)

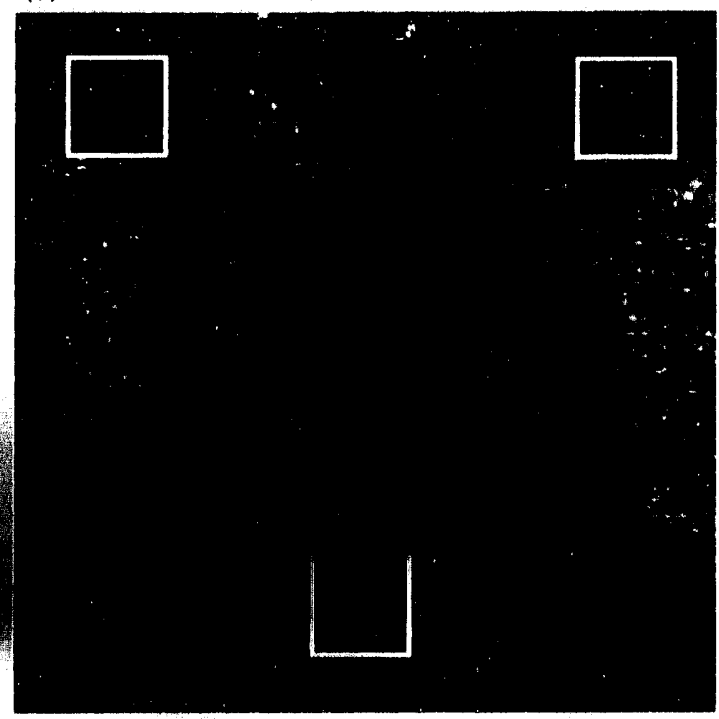

\section{Supervised Leaming}

An object-oriented framework for supervised learning using statistical pattern recogennition techniques and neural networks was formalized this vear. The framework consists of two claseses for manipulating databases for supervised learning; feat turesedertionalgerithms ' forevaluating and severting useful fentures for solving classification problems; and two now classifiers, a nearesteneightor classifier and a probabilistic neural network. It

Most of the supervised kearning algorithms in VISION (operate on a few data structures refered to as training and patterns tables. A 'training table' is a data structure that asseciates a label (typically a symbol) meant to represint the nome of a cattegory of patterns, with a collection of objerts (an instance of a collection-(bijert claks, in most casses). $A$ patterns table' is a sprecial kind of training table where the objerts in the collection are constriained to be fenture

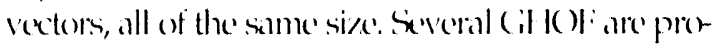
vided for pertorming transformations an these data structures. For example, in a typical application, we might start by ereating a training table that kerpes track of file namess with the original medsurements

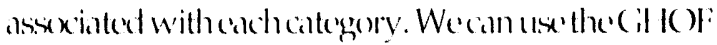
maptable to create a patterns table with the actual featurevecters tobeused by the karning algerithms. Consider the following example,

(selyfiles (moke-training-table :mulc'("ml" "m2" ...) :f(mulk'("fl" "i2" ...))

$\Rightarrow$ thining-tab)

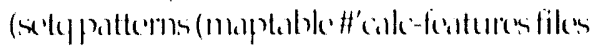

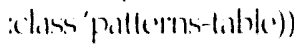

pollernstalle. 
In this example, we assume that the function calc-features has already been defined, such that given a file name, it reads the file, calculates the appropriate features, and returns a feature vector. The function maptable takes care of applying this function to every file name in the original training table and producing a new table with the actual feature vecters. The lambda expressions are very useful for prototyping functions like calc-features in order to try different kinds of features for the learning algorithms. Also, there is no need to store all of the initial raw data read from disk (which could be a serious problem with large databases), since only the final results (the feature vectors) are kept in memory.

Oncean initial set of features has been calculated, it is typically evaluated using one of the several featureselection algorithms in VISION, in order to find the best set of features that separate the $\mathrm{N}$-dimensional feature space. These features are then used to train one of the several classifiers in VISION, including a back-propagationneural network. ${ }^{15}$ These techniques have been successfully used for detecting and locating buried mines using dual-band, infrared sensors (see Fig, 4).16,17

\section{Future Work}

The main goal for $\mathrm{FY}-93$ is to complete the documentation for VISION 18 to make its capabilities more accessible to the LLNL community. We are also seeking technology transfer opportunities that will allow us to further expand our technology base in computer vision and pattern recognition. One organization from Pacific Gas and Electric Company is currently very interested in using VISION as its internal prototyping environment for applications in pattern recognition. We also expect current projects using VISION to contribute new algorithms and capabilities.

\section{Aclonowledgements}

The authors want to acknowledge the contributions made to VISION during FY-92 by Robert K. Johnson, Sailes Sengupta, Robert J. Sherwood, Paul C. Schaich, and William J. Maurer.

1. G.L. Steele, Jr., Commun Lisp: The Language, 2nd ed., Digital Press (Burlington, Massachusetts), 1990.

2. J.A. Lawless and N.M Miller, Understanding CLOS, The Common Lisp Object System, Digital Press (Burlington, Massachusetts), 1991.

3. S.A. Keene, Oljict-Oriented Programming in Common Lisp, Addison-Wesley (Reading, Massachusetts), 1989.

4. J.E. Hernande'̌, C.A. Clark, and S. Lu, "Computer Vision," Engintering Resemd, Development, and Tech- nology, Lawrence Livermore National Laboratory, Livermore, California, UCRL 53868-91, 8-5(1992).

5. J.E. Hernandez,S. Lu, R.J. Sherwood, G.A. Clark, and B.S. Lawver, A Signal and lmage Proxessing ObjectBased System Using CLOS, Lawrence Livermore National Laboratory, Livermore, California, UCRL-JC-108409 (1991).

6. N.K. Del Grande, G.A. Clark, P.F. Durbin, D.J. Fields, J.E. Hernandez, and R.J. Sherwood, "Buried Object Remote Detection Technology for Law Enforcement," Proc. SPIE Orlando '91 Symmosium (Orlando, Florida), (April 1-5, 1991).

7. W.J. Maurer, F.U. Dowla, and S.P. Jarpe, Seismic Event Interpretation Using Self-Organizing Neural Networks, L.awrence Livermone National Laboratory, Livermone, California, UCRL-JC-108630 (1992).

8. B. Myers, D. Giuse, R. Dannenberg, B. Zaden, D. Kosbie, E. Pervin, A. Mickish, and P. Marchal, "GARNET," IEEE Computer Magazine 11,71 (1990).

9. R.J. Johnson, T.W. Canales, D.L. Lager, C.L. Mason, and R.M. Searfus, "Interpreting Signals with an Assumption-Based Truth Maintenance System," Proc. SPIE - The International Society for Optical Engineering 786, 332 (May 1987).

10. J.E. Hernandez, Higher-Order Generic Functions for CLOS, Lawrence Livermore National Laboratory, Livermore, California, UCRL-JC-109776 (1992).

11. R. Haralick and L. Shapiro, Computer and Robot Vision, Volume 1, Addison-Wesley (Reading, Massachusetts), 1992.

12. G. Colemar and H. Andrews, "Image Segmentation by Clustering," Proc. IEEE 67 (5), (May 1979).

13. T. Young and K. Fu, Handbook of Pattern Recognition and Image Processing, Academic Press Inc. (San Diego, California), 1986.

14. D.F. Specht, "Probabilistic Neural Networks," Neural Neturorks 3, 109 (1990).

15. E.M Johansson, F.U. Dowla, and D.M. Goodman, Backpropagation Learning for Multi-Layered Feed-Forward Neurnl Networks Using the Conjugate Gradient Method, Lawrence Livermore National I aboratory, Livermore, California, UCRL-JC-104850(1991).

16. M.R. Buhl and J.E. Hernandez, Dual-Band, Infrared Buried Mine Detection Using A Statistical Pattern Recognition Appronch, Lawrence Livermone National Laboratory, Livermore, California, in preparation.

17. J.E. Hernandez, M.R. Buhl, and S. Sengupta, Detecting and Locating Buried Mines from Dual-Band IR Data: A Pattern Recognition Approach, Lawrence Livermore National Laboratory, Livermore, California, in preparation.

18. J.E. Hernandez, Using Vision, Lawrence Livermore National Laboratory, Livermore, California, UCRLMA-112337-DRAFT (1992). 


\title{
Biomedical Image Processing
}

\author{
Laura N. Mascio
}

: $\quad$ Defense Sciences Engineering Division

Electronics Engineering

We have developed a bio-imaging application for a genetics study and have made advances in projects related to automated fluorescence, microscopy, and mammography.

\section{Introduction}

In FY-92, we used funds from a small grant to make contributions to several biomedical research projects, including (1) colony filter analysis for genetic studies; (2) the human genome project; and (3) the detection of microcalcifications in digitized mammography.

\section{Progress}

\section{Colony Filter Analysis for Genetic Studies}

We have made progress in the automation of quantitative colony filter analysis (CFA), an important and versatile tool used by biologists for a variety of research goals. One application is to pinpoint interesting regions in human DNA so that more highly detailed analyses, such as sequencing, can be applied directly to these regions. Another goal is to very precisely determine the expression patterns of a gene. Using these patterns for comparison can provide a measurement of the genetic differences between distinct groups, such as male vs female, diseased persons vs non-diseased persons, or young persons vs old persons. One of the many other designs for a CFA experiment can yield the location of a certain DNA sequence, or gene, along a chromosome.

Because of its versatility, the CFA is a po iverful tool in today's genetics studies. Also because of its versatility, however, the analysis is highly complex, and automating this analysis is a technical challenge. One format for the data is an array of 18,000 radioactive data spots generated from a robotically prepared $2(1)-\mathrm{cm}-x-2()-\mathrm{cm}$ filter paper. Each of the 18,000 spots contains a signal of importance, although many signals may not be visible when imaged, and some are even difficult to de- tect computationally. When the filter paper has been imaged and digitized, it can form a data set up to $23 \mathrm{Mb}$ in size.

To automate the quantitation and location of each of the 18,000 signals, we first developed an image-processing algorithm that locates the spots that are detectable, and then predicts the location of those that are not. This algorithm and its platform (SCIL-Image) are capable of handling $23 \mathrm{Mb}$ of original data plus 4 to 5 times that for intermediate results.

Morphological image processing is the prominent methodology used in the automated CFA tool. The maximum (gray-scale dilation) and minimum (gray-scale erosion) operators are used in various combinations to provide background information, as well as texture or frequency information, for detecting the DNA colonies. These methods are documented thoroughly, and outlined briefly in Fig. 1. Once the algorithm has detected all spots, the image may be rotated so the colony array is aligned with the image. Then, long, thin, maximum filters are used to 'smear' the dots, first horizontally, and then vertically. The intersection of the smearing lines predicts the location of undetected spots. The grid is then rotated to fit over the original data. The rotated data cannot be used, because we are interested in quantifying the colonies. The affine transform that performs the rotation uses interpolation methods to assign each pixel a new value in the rotated image.

Next, we use the smearing lines to form a dynamic grid (non-uniform) over the data set, so that each grid square contains only one DNA colony. This grid provides the framework by which each DNA colony can be assigned a coordinate position. That is, while it is trivial to know the pixel coordinates of a spot, it is much more useful and difficult to know its grid position. The assignment of coordinates to the grid squares is not as trivial as 


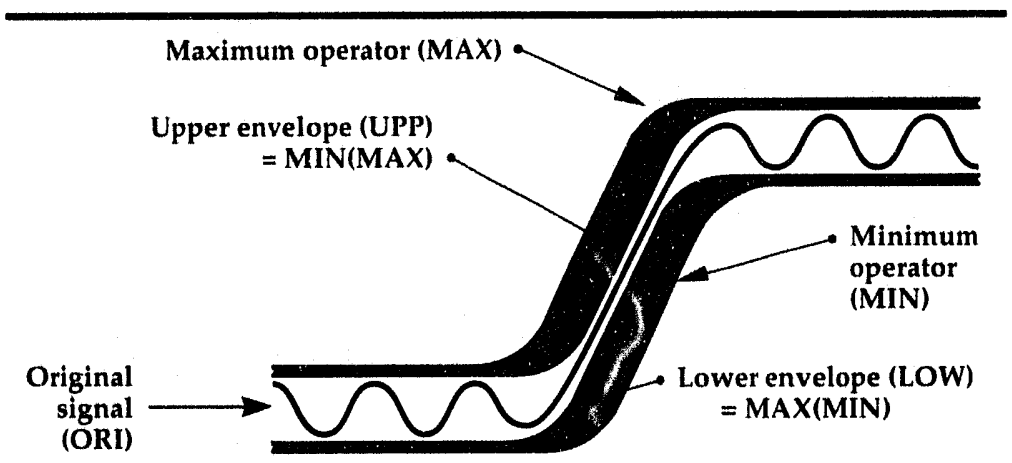

Figure 1. Maximum and minimum filters combined for powerful image processing. Shown is a one-dimensional signal and the effect of various max-min operator combinations. For the colony filter application, the lower envelope is used to estimate background values, so they can be subtracted before quantitation of the colonies. $A$ low-detail image is computed, so that the image-intensity characteristics can be sim plified before the high-detall image is computed. The high-detall image ylelds texture information, so the 'spots' formed by the colonies can be detected.

Figure 2. An example of a small filter with computer-generated grid-lines separating the objects. Each grid square must be assigned a grid coordinate, so that the results from each grid square can be related to the others.

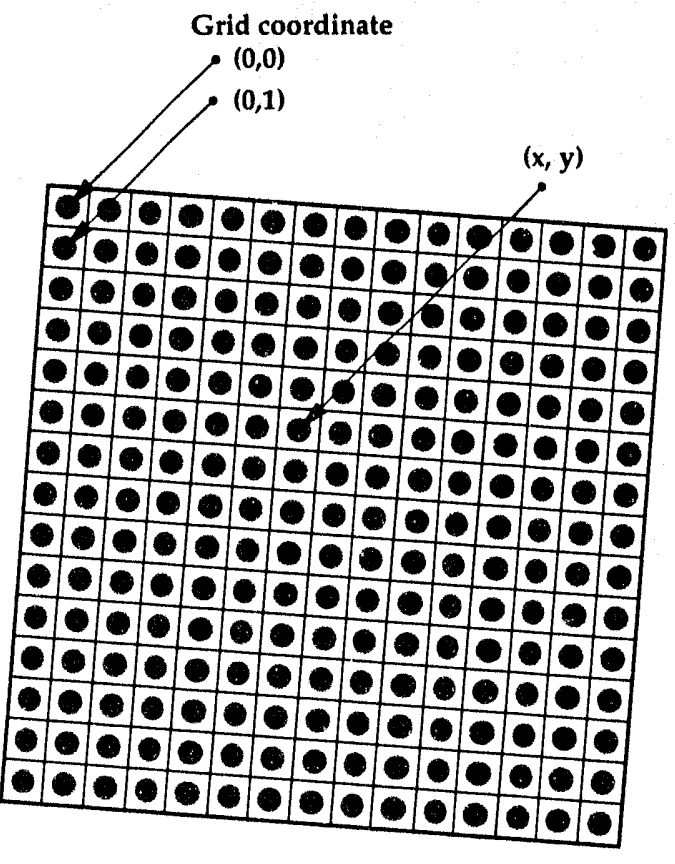

the obvious solution, because the grid is not necessarily square with the image, and in addition, the grid is not uniform (see Fig. 2).

Once each spot is localized and assigned grid coordinates, the total integrated intensity of the signal in each grid square is summed. This intensi- ty measurement must account for (subtract) global and local background intensities, which would act as a 'de offset' to the signal. After subtracting the background, any intensity from a surrounding signal must also be removed. Intensity values for each grid square can be reported, or queried individually by the investigator.

Upon further interaction with biomedical scientists, a final application can be customized for use with their data-acquisition routine.

\section{Automated Fluorescence Microscopy for DNA Mapping (Human Genome Project)}

We have undertaken the reconstruction of an automated fluorescence microscope. The equipment needed to construct this instrument, i.e., microscope, cooled CCD camera, automated filter wheel, automated stage, and controlling computer software, has been assembled, and the controlling software is nearly complete. We will use this instrument and a software application previously developed to demonstrate the potential to automate DNA probe mapping in metaphase chromosomes as a part of our human genome project.

\section{Detecting Microca!cifications in Digitized Mammograms}

We have made significant contributions to a research effort for early detection of cancer-warning signals in mammograms. This project is one that makes use of existing, but under-used equipment from nondestructive evaluation of weapons parts, i.e., a high-resolution digitizer for radiographs. By digitizing radiographs of breast images, computational methods, such as the morphological methods described in Fig. 1, can be used to analyze mammograms and to highlight areas that may require a radiologist's attention. The aim of this project is to hedp reduce the number of missed diagnoses of cancer in cases where warning signs exist on a mammogram.

1. P.W. Verbeek, H.A. Vromman, and L.J. Van Vliet, "Low Lewel Image Processing by Max-Min Filters," Sigrmal Prociss. 15, 249)(1988). 


\title{
Multisensor Data Fusion Using Fuzzy Logic
}

\author{
Donald T. Gavel \\ Laser Engineering Division \\ Electronics Engineering
}

We have developed an expert system based on fuzzy logic theory to fuse the data from multiple sensors and make classification decisions for objects in a waste reprocessing stream. Fuzzy set theory has found successful application in a number of decision and control applications ir recent years. We have found that a fuzzy logic system is rather easy to design and train, and that with proper training, classification accuracy is quite high. We performed several tests sorting radioactive test samples using a gamma spectrometer to compare fuzzy logic to more conventional schemes.

\section{Introduction}

The Department of Energy (DOE) has an urgent need for the development of waste processing and cleanup technologies. Over the past few years, the Advanced Process Technology Program at Lawrence Livermore National Laboratory has been developing roboticsand autor:ation technology to support cleanup and reclamation efforts. In our Interactive Controls Laboratriry, we have developed a sensor-based robot systern for material sorting tasks.

Robotic sorting of materials in a waste stream has been largely motivated by the DOE cleanup needs. A large fraction of the buried radioactive waste must be dug up and repackaged becausecontaminants are leaching into underground water tables. Hazardous waste stored in barrels at local sites must be resorted, according to federal guidelinesi, into categories of high-level, low-level, transuranic, and mixed waste, and disposed of accordingly. Certain materials, such as lead and stainless steel, can be reclaimed after being cleaned of radior active contamination. Low-level and mixed radioactive waste must be sorted into categrories, such as burnable or vitrifiable, for later volume reduction and storage. Using robots instead of radiation-suited workers reduces the risk to humans, and also improves the reliability and speed of operation.

Weapons dismantlement is now another important issue. Technologies neeet to be developed to handle the waste materials derived from dismantlement. In particular, recycling of depleted uranium alloys has historically not been done, but new federal guidelines will require it. The recycling of alloys $\mathrm{U}-\mathrm{Ti}$ and $\mathrm{U}-\mathrm{Nb}$ will require segmentation and tracking to prevent cross-contamination.

\section{Robot Sorting System}

We have assembled a demonstration robotic waste sorting and classification system (Fig. 1). This autormated workcell consists of a IPUMA 56() articulating robot arm, a machine vision system, a conveyor system, a suite of remote sensors, and a hierarchical computer control system that $\mathrm{cos}(\mathrm{r} d \mathrm{dinates}$ the activities within the workcell. A network of computers located within the laboratory allows real-time control

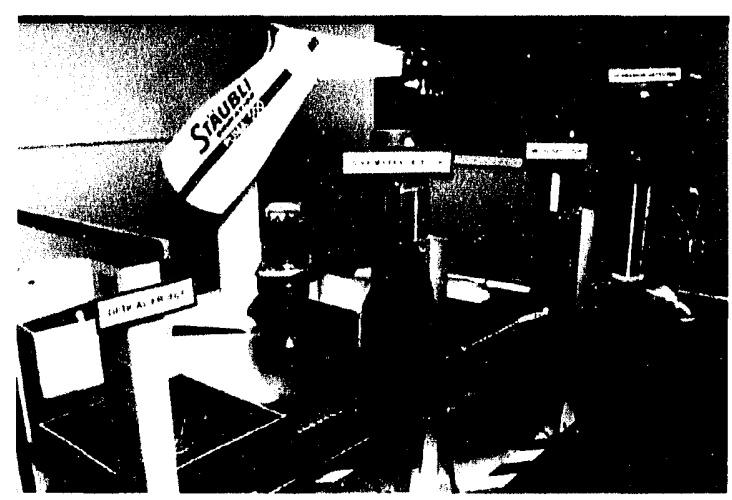

Figure 1. Interactive Controls Laboratory at Lawrence Livermore National Laboratory. The scrap conveyor is shown in the foreground along with the sensors used for material characterization. The PUMA robot arm with its wrist force/ torque sensor is in the background. Not shown is a stereo camera pair mounted on the ceiling. 


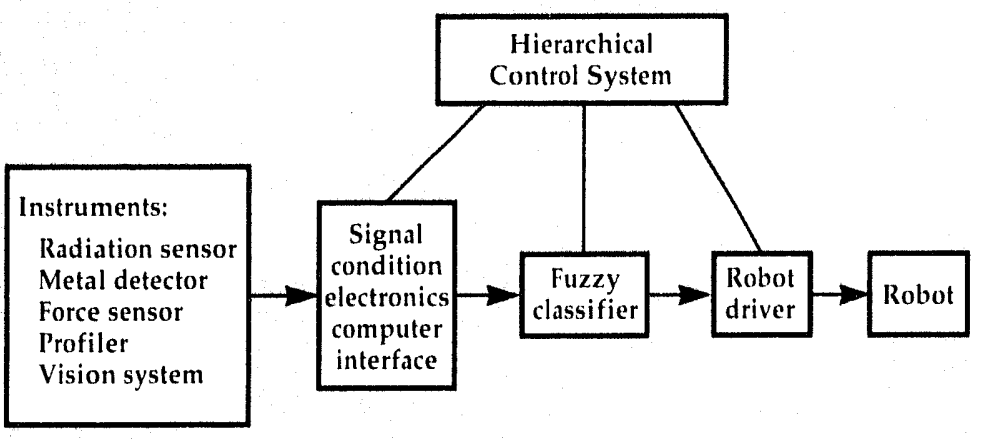

Figure 2. Block diagram of the robot control system. Input signals from various sensors are processed to determine the robot's next course of action. The fuzzy classifier categorizes the incoming material and provides this information to the robot driver.

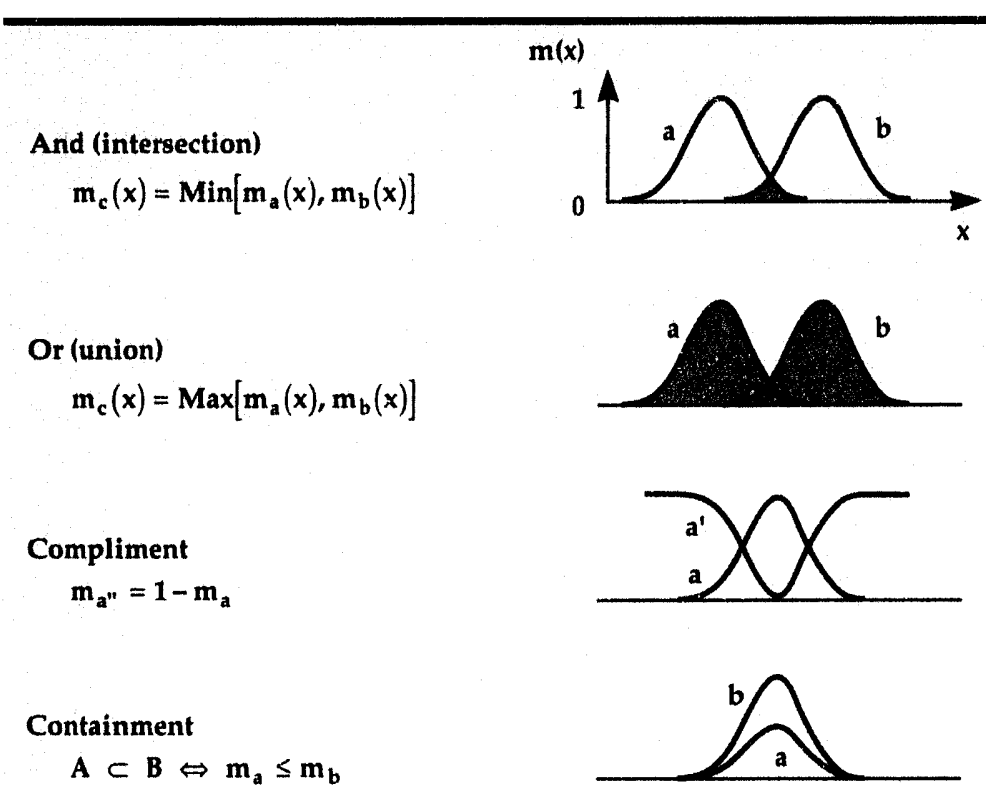

Figure 3. Operations on fuzzy sets. $x$ is a measured quantity. The inembership function $m_{t \prime}(x)$ defines the boundaries of the fuzzy set $(x$. Membership in ordinary sets take on values in $\{0,1\}$, whereas fuzzy sets allow membership in the continuous range $[0,1]$.

of the workcell through a three-dimensional graphical interface. Remote telersperation of the robot arm is supported, with 'telepresience' information such as contact forces and steree vision fed back to the operator. 1,2 Autonomous sensisor-directed operation is be ing develeped to handle difficult unstructured problems such as the scrap-sorting application described in this report.

\section{Fuzzy Set Classifier}

Robotic sorting of materials having unknown size, weight, and composition is a very different task from that of the usual picking and placing of known components in typical assembly lineoperations. The scrap materials must first be scanned by sensors lecated upstream in the conveyor system. The robot control system must then decide what action to take basect on the sensory inputs. We have develeped an expert system based on fuzzy logic therry to fuse the data from the multiple sensers and make classification decisions. Figure 2 shows a blexk diagram of the robot control system. Sonsing instruments include an optical profiler, gamma spectrometer, metal detector, force sensor (for weight), and machine vision system. From these measurements, we can infer size, density, metal content, and radiation content. Then, using the rule base, we can decermine if the object is lend, steed, plastic or other light materials, or radiation-contaminated versions of thewe. The rule base consists of linguistic inference statements such as:

(if weight is heavy and size is small then density is high)

(if density is high and metal content is high then object is lead).

These rules are derived from experience by human operators using detectors and a set of known test items. These rules can be coded into a computer so long as the computer has an exact definition of terms like "heavy" and "high." As humans, we asseciate vaguely defined properties with these terms. To use this same idea of vagueness in a rigorous mathematical context, a theory of fuzry sets has been developed. 'This therry introduces a new type of set, called a fuzzy set, which generalizes the concept of an ordinary set. Fuzzy set operations, analogrous to the logicaloperations on ordinary sets, aredefined accordingly (see Fig. 3). It is then a simple matter to implement a fuzzy legic inference engine on a computer. A fuzzy logic system takes input data from an experiment, applies the rules in its rule base, and comes up with conclusions. The output is a list of possible conclusions along with their degrees of confictence. If a hard decision must be made, as is the case in the wastesorting application (where the robot arm is instructed to place objects in various waste streams), then the conclusion with maximum confidence is taken.

\section{Experimental Results}

We performed experiments using gramma spextrometer data to compare the furzy legic inference technique to a detection threshold methert in categerrizing radiation sources. In thisexperiment, fourspectral bands resiolvable by the sexdium indicte detector were selected to correspend to strong emission lines of americium 241 (5) to $70 \mathrm{keV}$ ), thorium 232 (210 to 27() $\mathrm{k}(\mathrm{V})$ ), cesium $1.37(6(x)) \mathrm{to}, 730 \mathrm{k}(\mathrm{V})$ ), and cobalt $(x)$ (1270) to 14.30 keV), respertively. Howerer, since the emission spectra for these sources overlap, there is a 
Table 1. Results from an isotope identification experiment.

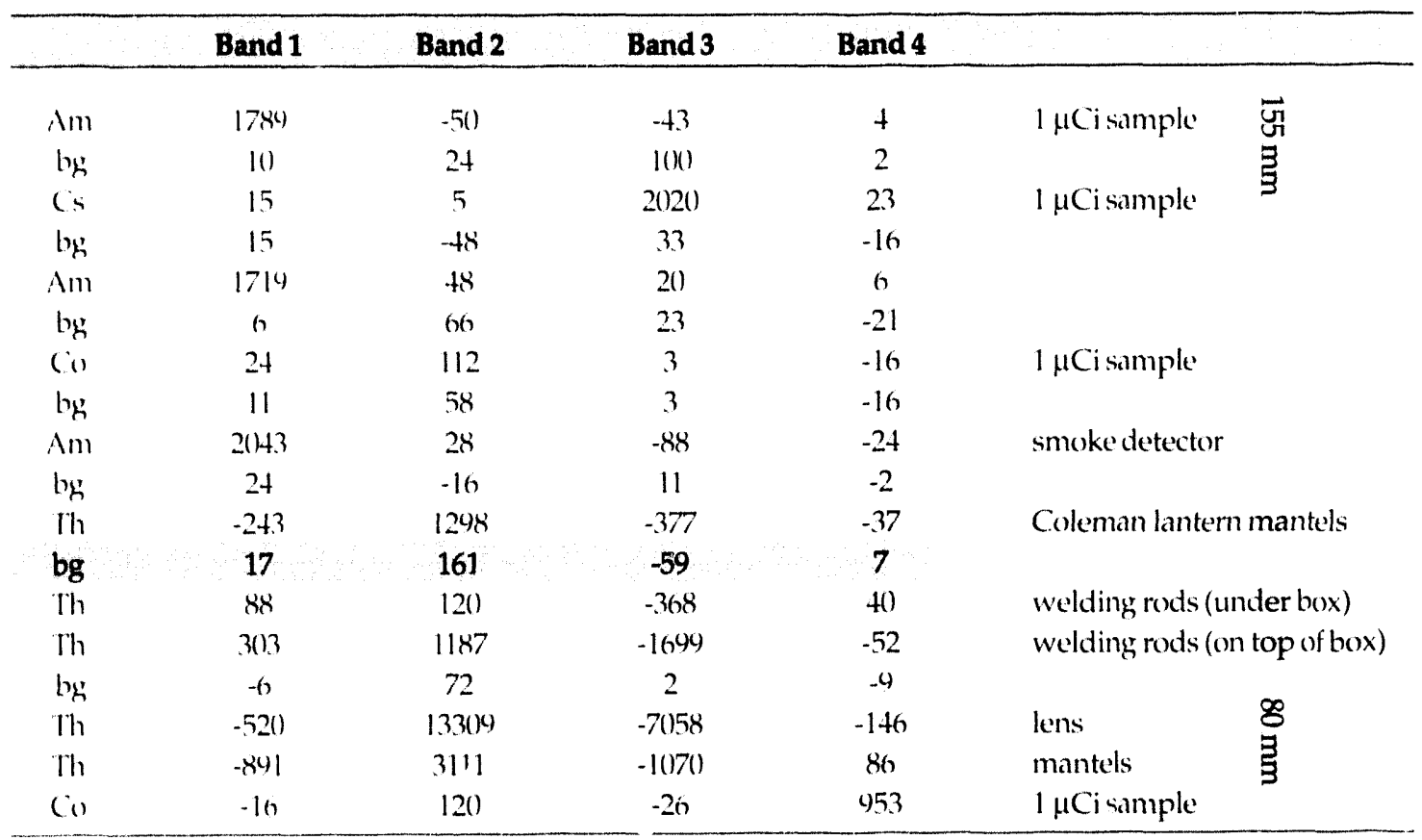

The highlighted area indicates where the threshold algorithm with a $10(0)$-count threshold produces a false alarm: background (bg) misidentified as thorium 232 (Th). The fuz/y logic system, relying more on pattern recognition, correctly indicates this as backgresund radiation, i.e., no source present. some 'counts' are negative because of data preconditioning and normalization.

pessibility that information conceming the presence of thorium, for example, is present in the cesium band, and wom. Therefore, information useful in the categorization of radioisotopes is containet in the paltem of the coumts, not just in the counts per individual band. The rules in the fuzzy rule base were set accordingly. For example:

(if band 4 is high or (band 4 is high and band 2 is high), then source is cobalt $(x)$ ).

Results from one test are shown in Table 1. We texited simple sources lexated from s() to 150$) \mathrm{mm}$ from the detextor, and alsow made an expual number of tests with no source present. The furey logic system conrectly identified the isotopes with lo(x)" accuracy, while the theshold system had only se" "accuracy (one miss and one incorrect classification) with a $2(x)$ count threshold, and $72 "$ " accurioy (three incorrect classifications and two false alarms) with a I (X)toount threxihold. As the sources are separated from the detectorby larger distances, the signal becomes weak(r, so the sensor and furas le gric system bey in to fail to detect the radiation. However, even with weak signals, the isotepe signature is often still present. Wese't up the furey le gic system to guess the ixotepe: even if the radiation count was low: At average xeparations of roughly $30(0) \mathrm{mm}$, the threshold system was failing nearly lox"', of the time, while the fuedy classifier was

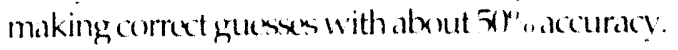

\section{Future Work}

Sorting and classification of materials will be a crucial task in the weapons dismantlement process. Special nuclear materials resulting from dismantlement need to be identified and tracked by an automated system to prevent unauthorized diversion from the recycle stream. Depleted uranium alloys should be segmented from each other to prevent cross-contamination. We see this as a future growth area for multisensor fusion and fuzzy classification systems such as the one we have developed.

1. D.T. (ivel and S.-y. I u, "Telerobuticand Machine

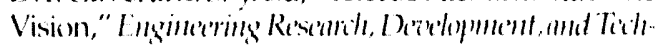
nolegy, l awrence Lisermore Nationall I aboratory, livermore, California, UCRL -53868-91,4-6) (1492).

2. A. Dougan, D.T. Ciavel, D. Ciustareson, M. Ilolliday, R. Hurd, R. Johneson, B. Kettering, and

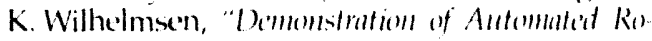
botic Workiell for Hazardous Woste Characterization." submitted to 199.3 HEEE Intermational Cont. Robotissand Automation (Atlanta, Cieorgia), (May | (x)3).

3. I.A. Tadeh, Int Comtrol $8,338(1965)$. 


\section{Adaptive Optics for Laser Guide Stars}

\author{
James M. Brase, \\ Kenneth Avicola, \\ Donald T. Gavel, and \\ Kenneth E. Waltjen \\ Laser Engineering Division \\ Electronics Engineering
}

\author{
Horst D. Bissinger \\ Energy Systems Engineering \\ Mechanical Engineering
}

We are investigating advanced concepts in adaptive optics $(\mathrm{AO})$ systems and developing a comprehensive analysis and modeling capability to predict the performance of $\mathrm{AO}$ systems. In FY-92, we demonstrated the generation of a $\mathrm{Na}$ guide star and verified our models of its formation. We have made the first Hartmann-sensor wavefront measurement from a Na guide star, and evaluated its potential as a reference for a closed-loop AO system.

\section{Introduction}

Turbulence in the atmosphere blurs images seen in ground-based telescopes and places a severe limit on their angular resolution. Typical atmospheric blurring is so severe that even a $10-\mathrm{m}$ telescope has no better resolution than a small 8-in. telescope, despite the fact that the larger instrument gathers far more light.

There are two methods for gaining dramatically improved resolution. The first is to go above the atmosphere, as did the Hubble Space Telescope. This approach has the additional advantage that regions of the spectrum such as the ultraviolet, which cannot penetrate the atmosphere, are accessible. However, going into space is expensive and inherently less flexible than observing from the ground. The second alternative is to use a tech- nique called 'adaptive optics' (AO) to improve resolution for ground-based telescopes. We are investigating advanced concepts in AO systems and developing a comprehensive analysis and modeling capability to predict the performance of AO systems.

AO systems have been demonstrated for astronomical applications. 1 These systems use a bright natural star as a reference to correct the dimmer astronomical object. One of the major problems with applying $\mathrm{AO}$ to astronomy is the scarcity of natural stars close enough and bright enough to serve as references. Our approach to solving this problem is shown in Fig. 1. We will use the copper-vapor pumped dye laser system, developed for laser isotope separation at Lawrence Livermore National Laboratory (LLNL), to illuminate a small circular area of the atmospheric sodium lay-

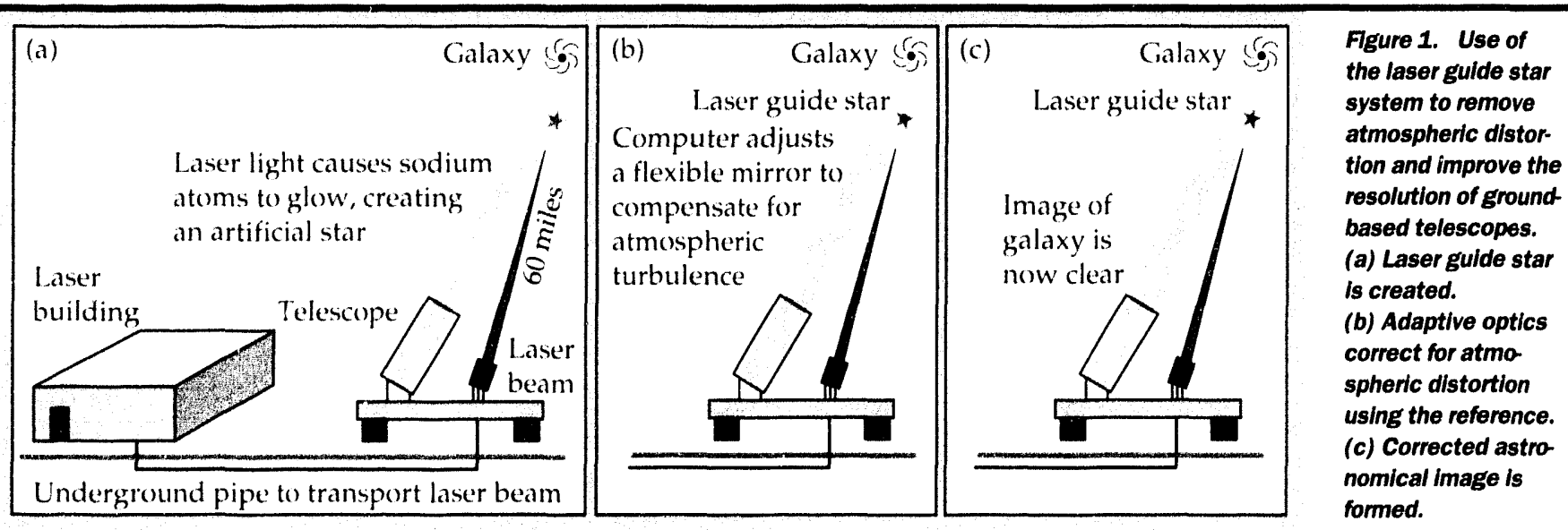



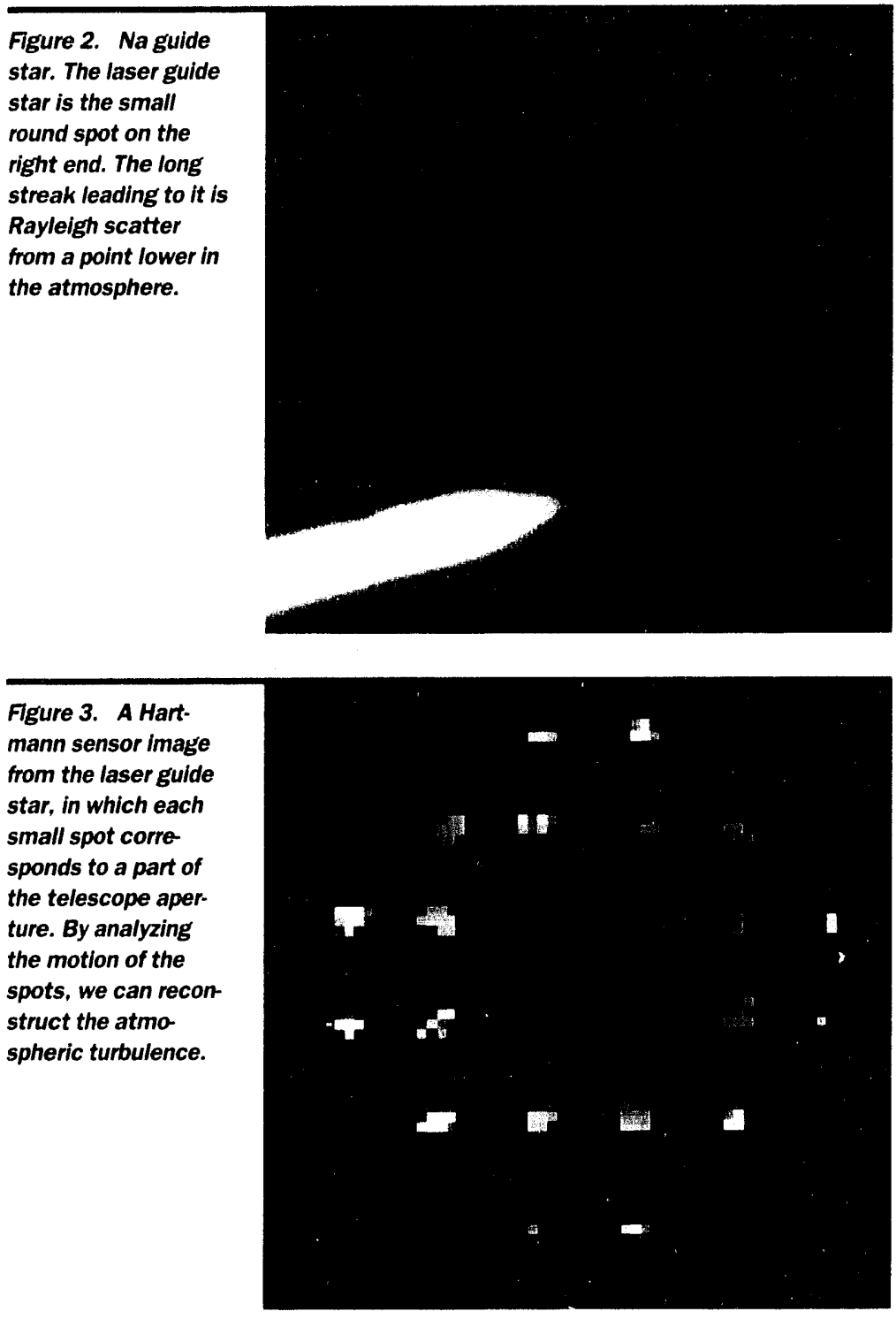

Figure 4. The predicted sodium emission intensity (solid line) vs the experimental measure ments (squares) from the Na guide star at several laser power levels.

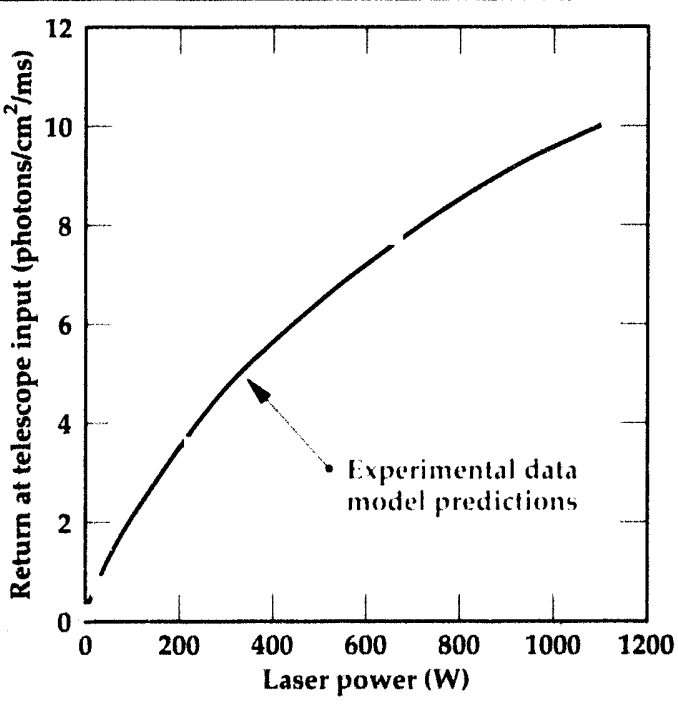

erat a height of about $100 \mathrm{~km}$. When the laser is tuned to the proper wavelength, the sxtium will glow and protuce a point-like reference source. An observing telescope on the ground measures in detail the light coming from this 'laser guide star' and, with the aid of a computer, deduces what distortions have been placexi on the wavefront by atmospheric turbulence. The computer then calculates the corrections to be applied to a deformable mirror in the optical train of the telescope to correct for the turbulence. The light from a nearby astronomical object is also corrected by the deformable mirror se that an improved image is formed.

The basic technologies for laser-guide-star $\mathrm{AO}$ systems have been demonstrated over the past ten years. 2,3,4 Success with $\mathrm{Na}$ guide stars has been limited by the lack of an appropriate laser. However, the LLNL copper-vapor pumped dye laser is well-suited for the demonstration of astronomical laser guide stars. It has more than enough power at the Na wavelength $(1.5 \mathrm{~kW}$ at $589 \mathrm{~nm})$, excellent reliability, and high beam quality. We are performing a series of feasibility experiments on laser guide stars using this laser. From the data obtained in these experiments, we will be able to design a smaller and more economical system optimized for use at an astronomical observatory. Our long-term goal is to establish a technology base in $\mathrm{AO}$ that will allow us to implement a system for a large astronomical instrument such as the 1()-m Keck Observatory telescope.

The laser guide star experiments at LLNL are being done in two phases. In the first, which began in July 1992, we have generated a $\mathrm{Na}$ guide star (Fig. 2) and have measured its intensity and motion. ${ }^{5}$ In the second phase, currently underway, we are developing an $A O$ system to demonstrate closed-loop correction of an astronomical object with a $\mathrm{Na}$ guide star."

\section{Progress}

In FY-92, we demonstrated the generation of a Na guide star and verified our models of its formation. We have made the first Hartmann semsor wavefront measurement from a $\mathrm{Na}$ guide star, and evaluated its potential as a reference for a closed-loop AO system.

\section{Wavefront Sensing}

An important part of an AO system is the sensor that analyzes the laser guide star wavefront in real time. ()ver the past year, we have developeda new high-sped thartmann wavefront sensorer ca- 
pable of measuring local wavefront slopes at one thousand frames per second. In a recent series of experiments, we made the first Hartmann-sensor wavefront measurements of a Na laser guide star. A typical Hartmann image is shown in Fig. 3. The motion of individual spots in these images is analyzed to estimate local wavefront slopes. These slopes are ultimately integrated into the wavefront phase distribution, which is used to control the deformable mirror. We are in the process of analyzing this preliminary data and performing more experiments to characterize the performance of this system.

We have also performed a series of wavefront sensing experiments using natural stars, to determine requirements for $A O$ systems at $L L N L$. These experiments will be expanded to include the University of California's Lick Observatory on Mt. Hamilton as the first step towards implementation of an $\mathrm{AO}$ system there.

\section{Analysis and Modeling}

Our long-term goal is to develop laser guide star systems for 10-m-class telescopes like that of the Keck Observatory. The initial development, however, will take place on smaller telescopes both at LLNL and at Lick Observatory. It is vital that we use computer simulations to understand the scaling of the results from our demonstration experiments, to what we should expect from large astronomical telescopes. The initial experiments will allow us to validate our simulations, so that we can have a greater degree of confidence in the results for 10-m telescopes.

Some problems that will arise on large telescopes will not be evident in our smaller systems. For example, as the telescope gets large, a single laser guide star can no longer be used to correct the entire aperture, because of the finite height of the laser guide star. Multiple laser guide stars must be generated to accurately correct the images. A complete simulation will allow us to develop these techniques even before we have access to a large telescope.

$\mathrm{To}$ date, implementation of astronomical $\mathrm{AO}$ systems has been devoted mainly to system development. Very little actual astronomy with adaptively corrected telescopes has yet been done anywhere in the world. Because of the change in quality of the correction across the ficld of view and with changes in atmospheric conditions, stan- dard astronomical measurements such as photometry and spectroscopy may become more complicated. Our simulation tools will allow us to explore these problems before large-scale AO systems are designed.

One of our first tasks in model validation has been to compare the results of our initial laserguide-star experiments with the predictions of our simulations. The comparison of predicted Na emission intensity with the experimental measurements is shown in Fig. 4. The excellent agreement increases our confidence in other simulation results.

\section{Future Work}

In FY-93, we will demonstrate closed-loop AO correction of a small telescope at LLNL using a $\mathrm{Na}$ guide star. This demonstration experiment will require the wavefront sensing technology that we have developed. It will also allow us to verify our analysis and simulation tools. We are beginning to apply these techniques to a variety of new problems in highresolution imaging and beam control.

1. C. Rousset, J.C. Fontanella, P. Kern, D. Cigan, F. Rigaut, I?. Lena, C. Boyer, I? Jagourel, J.P. Gaffard, and F. Merkle, Astron. Astrophlyss. 230, L29(1990)).

2. R. Fugate, D. Fried, G. Ameer, B. Boeke, S. Browne, P. Roberts, R. Ruane, G. Tyler, and L. Wopat, Natur' 353 (September 12, 1991).

3. C. Primmerman, D. Murphy, D. Page, B. Zollars, and H. Barclay, Noture 353 (September 12, 1991).

4. C. Gardner and L. Thompson, Proc. IEEE 78 (11), $1721(199))$.

5. K. Avicola, J.M. Brase, J.R. Morris, H.D. Bissinger, H.W. Friedman, D.T. Gavel, C.E. Max, S.S. Olivier, R.W. I'resta, D.A. Rapp, J.T. Salmon, and K.E.

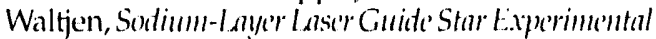
Re'sults, Lawrence Livermore National Laboratory, Livermore, California, UCRL-JC-111896 (1992).

6. C.E. Max, H.W. Friedman, J.M. Brase, K. Avicola, H.D. Bissinger, D.T. Gavel, J.A. Horton, J.R. Morris, S.S. Olivier, R.W. I'resta, D.A. Rapp, J.T. Salmon, and K.E. Waltjen, Design, Lanyout, and Early Re'sults of a Fensibility Experime'nt for Sodium-lanier Laser Contide Stur Aituptine Optics, Lawrence Livermore National Laboratory, Livermore, California, UCRI.IC-112162(1992).

7. R.K. Tyson, Principles of Aduptione Optics, Academic Press (Boston, Massachusetts), 1991. 
